\title{
A Modeling and Simulation Study of the Role of Suspended Microbial Populations in Nitrification in a Biofilm Reactor
}

\author{
Alma Mašić • Hermann J. Eberl
}

Received: 9 August 2012 / Accepted: 19 August 2013 / Published online: 5 December 2013

(C) Society for Mathematical Biology 2013

\begin{abstract}
Many biological wastewater treatment processes are based on bacterial biofilms, i.e. layered aggregates of microbial populations deposited on surfaces. Detachment and (re-)attachment leads to an exchange of biomass between the biofilm and the surrounding aqueous phase. Traditionally, mathematical models of biofilm processes do not take the contribution of the suspended, non-attached bacteria into account, implicitly assuming that these are negligible due to the relatively small amount of suspended biomass compared to biofilm biomass. In this paper, we present a model for a nitrifying biofilm reactor that explicitly includes both types of biomass. The model is derived by coupling a reactor mass balance for suspended populations and substrates with a full one-dimensional Wanner-Gujer type biofilm model. The complexity of this model, both with respect to mathematical structure and number of parameters, prevents a rigorous analysis of its dynamics, wherefore we study the model numerically.

Our investigations show that suspended biomass needs to be considered explicitly in the model if the interests of the study are the details of the nitrification process
\end{abstract}

Electronic supplementary material The online version of this article

(doi:10.1007/s11538-013-9898-2) contains supplementary material, which is available to authorized users.

\footnotetext{
A. Mašić

Centre for Mathematical Sciences, Lund University, P.O. Box 118, SE-22100 Lund, Sweden

Present address:

A. Mašić (\)

Eawag, Swiss Federal Institute of Aquatic Science and Technology, Überlandstrasse 133, P.O. Box 611, CH-8600 Dübendorf, Switzerland

e-mail: alma.masic@eawag.ch

H.J. Eberl

Biophysics Interdepartmental Program and Dept. Mathematics and Statistics, University of Guelph, N1G 2W1 Guelph, ON, Canada

e-mail: heberl@uoguelph.ca
} 
and its intermediate steps and compounds. However, suspended biomass may be neglected if the primary interests are the overall reactor performance criteria, such as removal rates. Furthermore, it can be expected that changes in the biofilm area, attachment, detachment, and dilution rates are more likely to affect the variables primarily associated with the second step of nitrification, while the variables associated with the first step tend to be more robust.

Keywords Biofilm $\cdot$ Mathematical model $\cdot$ Nitrification $\cdot$ Suspended biomass

\section{Introduction}

The study of microbial population and resource dynamics has a long history in Mathematical Biology. Traditionally, these models focus on suspended bacterial populations in well mixed reactors such as the chemostat. The basic mathematical theory for this type of models is relatively well established (Smith and Waltman 1995), but the field remains an active research area and the model framework continues to be extended to biologically, physiologically, and mathematically more complex systems, e.g. Fgaier and Eberl (2011), Mazenc and Malisoff (2012), Northcott et al. (2012), Peña Miller et al. (2012), Smith and Thieme (2012) as selected current examples. While the well stirred, completely mixed chemostat is an established reactor for laboratory experiments in microbiology and microbial engineering (Desharnais and Yiqi 2005; Villadsen et al. 2011), it is widely recognized that in many natural or engineered systems bacteria also, and often preferably, colonize surfaces and typically contain both suspended and sessile microbes. This is used in particular in environmental engineering for the design of biological wastewater treatment processes (Rittmann and McCarty 2001).

An early example of a mathematical model for a reactor with suspended and wallattached bacteria is Freter et al. (1983), which was later adapted for a microbially relatively simple system in a completely mixed reactor and analyzed in several papers, including Stemmons and Smith (2000), Ballyk et al. (2001), and Jones et al. (2003). In this model, it is assumed that the available surface area limits wall attachment and that the microbial layer on the wall is essentially a thin layer in which the bacteria experience the same growth conditions as the suspended bacteria in the liquid phase. In many real world systems, however, wall attached bacteria form relatively thick layers, up to several hundreds of microns or more, in which, due to consumption and diffusion growth promoting substrates such as nutrients or oxygen undergo concentration gradients, with the consequence that the bacteria in the inner layers of the film experience different growth conditions than the bacteria in the outer layers or in the suspended liquid phase. Consequently, this leads to spatially non-homogeneous species distribution. These microbial layers are commonly referred to as bacterial biofilms.

A mathematical modeling framework for biofilm processes was introduced in the 1980s in the engineering literature in the seminal paper Wanner and Gujer (1986). This model has been used since in numerous simulation studies of microbially complex multi-species, multi-substrate systems; see Chaudhry and Beg (1999) for an 
early overview, as well as Wanner et al. (2006). In this modeling framework, a biofilm is considered a homogeneous, one-dimensional layer on a surface, which grows due to the conversion of substrates into new biomass. The model constitutes a mixed hyperbolic-parabolic free boundary value problem for the microbial populations and the growth controlling substrates. Mathematically, this leads to rather involved models that are not easily accessible to mathematical analysis. A recent attempt to combine the Wanner-Gujer biofilm model with the Freter model of wall-attachment for the much simpler case of a single species, single substrate biofilm was made in Mašić and Eberl (2012). In this special case, the system can be formally reduced to a system of ordinary differential equations that could be studied qualitatively, at least partially, with elementary techniques. While this model is algebraically more involved than the Freter model, it was found that the qualitative behavior of both models is comparable. In the current study, we want to extend this to the microbially more complex multi-species, multi-substrate system that arises in wastewater treatment.

Bacterial communities are used in wastewater treatment for removal of harmful or undesired compounds, such as ammonium. In a well-mixed reactor with continuous in and outflow, the bacteria can grow as sludge in the water or as biofilms on suspended carriers. The carriers provide protected surfaces on which biofilms can grow and remain in the reactor without being exposed to the risk of washout. Nitrification, an aerobic process, takes place in waters containing no organic matter, where autotrophic bacteria convert ammonium to nitrate in two steps (Gray 2004; Rittmann and McCarty 2001): ammonium oxidizing bacteria (AOB) convert ammonium $\left(\mathrm{NH}_{4}^{+}\right)$to nitrite $\left(\mathrm{NO}_{2}^{-}\right)$and nitrite oxidizing bacteria (NOB) convert nitrite to nitrate $\left(\mathrm{NO}_{3}^{-}\right)$. For an influent with given characteristics, the performance of a continuous-flow stirred tank reactor (CSTR) that is augmented with suspended carriers for biofilm growth depends largely on operating conditions such as the dilution rate and the surface area available for biofilm formation. While this type of reactor is designed as a biofilm reactor (Moving Bed Biofilm Reactor, MBBR), and under the assumption that the conversion processes take place in the biofilm, suspended biomass cannot be avoided. Biofilm detachment and (re-)attachment of suspended biomass to the biofilm lead to a steady exchange between both modes of growth. Traditional mathematical models of this process include the biofilm only but neglect the presence and potential contribution of the suspended biomass (e.g. Mašić et al. 2010; Morgenroth et al. 2000). Our aim in this paper is to incorporate the suspended biomass by combining a CSTR model of nitrification with a biofilm model of nitrification. In contrast to the biofilm technology that we are considering, newer treatment processes, such as the Integrated Fixed-Film Activated Sludge (IFAS) process (Johnson et al. 2004), are designed with the interplay of sessile and suspended populations in mind. These naturally require models that combine the effects of both biofilm and suspended biomass. In our case, the suspended biomass is not a design feature of the reactor, but it is merely considered a "side effect" of the biofilm. Investigating the importance of this side effect is the focus of the present study.

Mathematical models for hybrid complex, multi-species/multi-substrate systems comprising biofilms and suspended biomass have been proposed in the literature before, including for the nitrification process; see Boltz et al. (2011, 2009a, 2009b, 2009c), Di Trapani et al. (2010a, 2010b), Fouad and Bhargava (2005), Kim et al. 
(2009), Mannina et al. (2011), Sen and Randall (2008a, 2008b), and Thalla et al. $(2009,2010)$. These earlier studies invoke one or more additional greatly simplifying assumptions to reduce the computational complexity of the overall model. Primarily, these assumptions are based on neglecting the heterogeneous distribution of biomass across the biofilm, and/or they use approximating empirical or heuristical relationships for the substrate flux between biofilm and liquid phase, instead of solving the underlying diffusion-reaction system that governs substrate concentrations and gradients in the biofilm. See Mašić (2013) for a more detailed overview and discussion.

In the model presented here, we do not include these simplifications, but we describe the biofilm by a full traditional one-dimensional model of Wanner-Gujer type that we embed in a lumped reactor mass balance for dissolved substrates and suspended bacteria. Overall, the resulting model is a system of ordinary differential equations that is coupled to a hyperbolic free boundary value problem with non-local non-linearities for the biomass distribution in the biofilm by a semi-linear system of second-order two-point boundary value problems for the substrates in the biofilm. The model is not easily accessible for rigorous analysis of the dynamic behavior. Therefore, it is studied in extensive numerical simulations.

Besides giving a description of the overall system behavior, we aim to answer the following questions:

- For given reaction parameters, how do the operating conditions of the reactor, expressed in terms of hydraulic load and colonizable surface area, affect the biofilm nitrification process qualitatively and quantitatively?

- What is the quantitative contribution of the suspended bacteria to the predominantly biofilm controlled nitrification process?

- Can a simpler model that considers only the biofilm but does not explicitly account for transformation processes in the suspended phase give a sufficiently good approximation of the process?

- What are the effects of attachment and erosion in the hybrid model?

- How do attachment rate, dilution rate, colonizable surface area, and erosion parameter affect the relative differences between the hybrid and the biofilm-only model?

- How well is the microbially complex system studied here described qualitatively by the much simpler previously studied single-species model?

\section{Mathematical Model}

\subsection{Governing Equations}

The main structure of our model originates in Jones et al. (2003), where substrates, floc forming suspended bacteria, and wall-attached bacteria are considered in a continuously stirred tank reactor for a single species system that is growth limited by a single substrate. We extended this model in Mašić and Eberl (2012), where we replaced the simpler Freter model for wall attached bacteria by the traditional 1D biofilm model from Wanner and Gujer (1986). We now introduce microbial complexity into the previous single species/single-substrate case by considering the nitrification process described in Elenter et al. (2007) for biofilms. 
In this multi-species multi-substrate model, bacteria are assumed to detach and become suspended, and suspended bacteria can (re-)attach to the biofilm. Biomass growth occurs through consumption of substrate, which is continuously supplied to the reactor. Substrates diffuse into the biofilm and form gradients due to diffusion and reaction. Bacterial cells may become inert matter through inactivation and endogenous respiration. The flow through the reactor washes out suspended biomass as well as substrates and products. Biofilms are assumed to homogeneously cover the colonizable surfaces on which they grow.

Starting point for the development of our model are mass balances for the dissolved substrates in a reactor with a completely mixed bulk phase,

$$
\dot{S}_{k}=D\left(S_{k}^{0}-S_{k}\right)-\frac{1}{V}\left(r_{k}\left(u_{A}, u_{N}, \bar{S}\right)+A D_{k} j_{k}(\lambda, \bar{S})\right), \quad k \in I_{S}
$$

with initial conditions

$$
S_{k}\left(t_{0}\right)=S_{k}^{0},
$$

where $I_{S}=\{\mathrm{O} 2, \mathrm{NH} 4, \mathrm{NO} 2, \mathrm{NO} 3\}$ is the index set, $S_{\mathrm{O} 2}(t)\left[\mathrm{g} / \mathrm{m}^{3}\right]$ denotes the oxygen concentration, $S_{\mathrm{NH} 4}(t)$ ammonium, $S_{\mathrm{NO} 2}(t)$ nitrite, $S_{\mathrm{NO} 3}(t)$ nitrate, and where $\bar{S}=\left[S_{\mathrm{O} 2}, S_{\mathrm{NH} 4}, S_{\mathrm{NO} 2}, S_{\mathrm{NO} 3}\right]$. The reactor parameters $D\left[\mathrm{~d}^{-1}\right], V\left[\mathrm{~m}^{3}\right]$, and $A\left[\mathrm{~m}^{2}\right]$ denote the dilution rate, the volume, and the colonizable surface area, respectively. The dilution rate can be related to the flow rate through $D=Q / V$, where $Q\left[\mathrm{~m}^{3} / \mathrm{d}\right]$ is the flow rate. The diffusion coefficients for the dissolved components are denoted by $D_{k}\left[\mathrm{~m}^{2} / \mathrm{d}\right], k \in I_{S}$. Table 1 gives the stoichiometric matrix from which the reaction rates $r_{k}$ can be expressed, which are also specifically written out in Appendix A. In essence, these reaction rates describe uptake or production of substrates by microbial activity. Using the same notation as in Mašić and Eberl (2012), the functions $j_{k}(\lambda, \bar{S}), k \in I_{S}$, are defined as

$$
j_{k}(\lambda, \bar{S})= \begin{cases}\frac{1}{D_{k}} \int_{0}^{\lambda} r_{k}\left(f_{A} \rho, f_{N} \rho, \bar{C}\right) d z & \lambda>0 \\ 0 & \lambda=0\end{cases}
$$

where $\lambda(t)[\mathrm{m}]$ is the biofilm thickness. The suspended biomass types $u_{A}, u_{N}$, and the biofilm fractions $f_{A}, f_{N}$ will be discussed below. Diffusion-reaction equations describe the dissolved compounds within the biofilm. Due to the disparity of the time scale for biomass growth and substrate consumption and diffusion (see Wanner et al. 2006), we follow the common practice in biofilm modeling to consider the equations at steady state,

$$
D_{k} C_{k}^{\prime \prime}(z)=r_{k}\left(f_{A} \rho, f_{N} \rho, \bar{C}\right), \quad 0<z<\lambda, k \in I_{S}
$$

with boundary conditions

$$
C_{k}^{\prime}(0)=0, C_{k}(\lambda)=S_{k},
$$

where $C_{\mathrm{O} 2}(z)\left[\mathrm{g} / \mathrm{m}^{3}\right]$ denotes oxygen concentration at biofilm thickness $z \mathrm{~m}$ from the substratum, $C_{\mathrm{NH} 4}(z)$ ammonium, $C_{\mathrm{NO} 2}(z)$ nitrite, $C_{\mathrm{NO} 3}(z)$ nitrate, and $\bar{C}=$ 
$\left[C_{\mathrm{O} 2}, C_{\mathrm{NH} 4}, C_{\mathrm{NO} 2}, C_{\mathrm{NO} 3}\right] . C_{k}(z)$ indirectly depend on $S_{k}$ through the boundary condition at $z=\lambda$ in (5). Note that the boundary conditions change with time through dependence on $S_{k}(t)$ and $\lambda(t)$, wherefore solutions to (4) are functions of time. Boundary condition (5) for $z=0$ imposes that the substratum is impermeable to the dissolved compounds.

Biomass exists in suspended and in biofilm form divided into three types: ammonium oxidizers $(A)$, nitrite oxidizers $(N)$, and inert matter $(I)$. In contrast to Elenter et al. (2007), we assume that heterotrophic bacteria are not present in the reactor, but consider a pure nitrifying system. Suspended biomass in the reactor is assumed to be completely mixed and described by the reactor mass balances

$$
\begin{aligned}
\dot{u_{A}}= & u_{A}\left(\mu_{A}(\bar{S})-D\right)-\alpha u_{A}+\operatorname{A\rho d}(\lambda) \lambda f_{A}(\lambda) \\
\dot{u_{N}}= & u_{N}\left(\mu_{N}(\bar{S})-D\right)-\alpha u_{N}+A \rho d(\lambda) \lambda f_{N}(\lambda) \\
\dot{u_{I}}= & \left(f_{X I}+\eta\right)\left(u_{A} b_{A} \cdot \frac{S_{\mathrm{O} 2}}{K_{A, \mathrm{O} 2}+S_{\mathrm{O} 2}}+u_{N} b_{N} \cdot \frac{S_{\mathrm{O} 2}}{K_{N, \mathrm{O} 2}+S_{\mathrm{O} 2}}\right)-u_{I} D \\
& \quad-\alpha u_{I}+A \rho d(\lambda) \lambda f_{I}(\lambda)
\end{aligned}
$$

with initial conditions

$$
u_{A}\left(t_{0}\right)=u_{A}^{0}, \quad u_{N}\left(t_{0}\right)=u_{N}^{0}, \quad u_{I}\left(t_{0}\right)=u_{I}^{0},
$$

where $u_{A}(t)[\mathrm{g}]$ denotes the suspended ammonium oxidizers, $u_{N}(t)$ nitrite oxidizers, and $u_{I}(t)$ inert matter. The details of the growth rates $\mu_{A}(t)\left[\mathrm{d}^{-1}\right]$ and $\mu_{N}(t)$ $\left[\mathrm{d}^{-1}\right]$ are found in Table 1 and in Appendix A. They comprise production of biomass due to substrate utilization, and loss of active biomass due to endogenous respiration and inactivation. $K_{A, \mathrm{O} 2}, K_{N, \mathrm{O} 2}\left[\mathrm{~g} / \mathrm{m}^{3}\right]$ are the half-saturation Monod coefficients for oxygen, $b_{A}, b_{N}\left[\mathrm{~d}^{-1}\right]$ are the decay rates, $f_{X I}[-]$ is the fraction of inert biomass produced by endogenous respiration and $\eta[-]$ is the anoxic reduction factor.

The biomass exchange process between biofilm and suspension occurs in both directions at different rates. As in Mašić and Eberl (2012), attachment is described by a linear function of the suspended biomass density with a constant attachment rate $\alpha\left[\mathrm{d}^{-1}\right]$. It is worth noting that a main difference between the attachment model used here and the one used in the Freter model with wall attachment in Jones et al. (2003) is that in the latter availability of free space can limit attachment, whereas in the former suspended biomass can attach to an existing biofilm, i.e. no limitation on the attachment rate is enforced.

In Wanner and Gujer-type biofilm models, the standard detachment rate $d(\lambda)$ $\left[\mathrm{d}^{-1}\right]$ is assumed to be proportional to the biofilm thickness with a constant erosion parameter $E\left[\mathrm{~m}^{-1} \mathrm{~d}^{-1}\right]$

$$
d(\lambda)=E \lambda
$$

For the description of the biofilm model, we follow the traditional Wanner-Gujer modeling framework from Wanner et al. (2006) and Wanner and Gujer (1986). In particular, it is assumed that within the biofilm the biomass density $\rho\left[\mathrm{g} / \mathrm{m}^{3}\right]$ is constant. Therefore, the distribution of biomass across the biofilm can be described by the volume fractions occupied by the different types of biomass which add up to unity. In 
the biofilm, new active biomass is produced due to utilization of substrates and transformed into inert biomass by endogenous respiration and inactivation, following the same kinetics as the suspended biomass. Production of new biomass leads to spatial expansion of the biofilm, where the expansion velocity is determined by the rate of biomass production. In the biofilm, spatial movement of biomass is described by the advection-reaction equation

$$
\frac{\partial f_{i}}{\partial t}=f_{i} \mu_{i}(\bar{C})-\frac{\partial}{\partial z}\left(f_{i} v\right), \quad 0<z<\lambda, i \in I_{b}
$$

with initial conditions

$$
f_{i}\left(z, t_{0}\right)=f_{i}^{0}(z)
$$

where $I_{b}=\{A, N, I\}$ is the index set for biomass, $f_{A}(z, t)[-]$ denotes the fraction of ammonium oxidizers at time $t$ and biofilm thickness $z, f_{N}(z, t)$ the nitrite oxidizers and $f_{I}(z, t)$ the fraction of inert matter. As above, the growth rates $\mu_{A}(t), \mu_{N}(t)$ and $\mu_{I}(t)$ can be found in Table 1 and in Appendix A. Note that the three volume fractions must satisfy

$$
\sum_{i \in I_{b}} f_{i}(z, t)=1
$$

In (11), the variable $v$ denotes the velocity of the biomass in the biofilm. It is computed from the reaction terms by

$$
v(t, z)=\int_{0}^{z} \sum_{i \in I_{b}} f_{i} \mu_{i}(\bar{C}) d z^{\prime} .
$$

The rate of change for the biofilm thickness $\lambda(t)[\mathrm{m}]$ is the sum of detachment, attachment, and biomass growth induced expansion. We have

$$
\begin{aligned}
\dot{\lambda}= & \int_{0}^{\lambda}\left(f_{A} \mu_{A}(\bar{C})+f_{N} \mu_{N}(\bar{C})+f_{I} \mu_{I}(\bar{C})\right) d z+\frac{\alpha}{A \rho}\left(u_{A}+u_{N}+u_{I}\right) \\
& -d(\lambda) \lambda\left(f_{A}(\lambda)+f_{N}(\lambda)+f_{I}(\lambda)\right) .
\end{aligned}
$$

\subsection{Some Remarks on the Mathematical Model}

Remark 2.1 (Complexity of the mathematical model) Equations (1)-(15) constitute a complex, hybrid mathematical model. To study it qualitatively with analytical methods is difficult, and even the analysis of the individual ingredients is challenging and often does not lead to insightful results, such as easy to apply stability criteria. Our model consists of a mass balance for the bioreactor, consisting of the eight ordinary differential equations (1), (6)-(8), (15) for bulk substrate concentrations $S_{\mathrm{O} 2}(t), S_{\mathrm{NH} 4}(t), S_{\mathrm{NO} 2}(t), S_{\mathrm{NO} 3}(t)$, suspended biomass $u_{A}(t), u_{N}(t), u_{I}(t)$, and biofilm thickness $\lambda(t)$. The nonlinearities in this mass balance are coupled to and require the solution of a one-dimensional biofilm model of Wanner-Gujer type. 
Table 1 Stoichiometric matrix

\begin{tabular}{|c|c|c|c|c|c|c|c|c|c|}
\hline \multirow{2}{*}{ Process $j$} & \multicolumn{4}{|c|}{ Dissolved compounds $k$} & \multicolumn{3}{|c|}{ Biomass components $i$} & \multirow{2}{*}{\multicolumn{2}{|c|}{ Process rate $P_{j}$}} \\
\hline & $S_{\mathrm{O} 2}$ & $S_{\mathrm{NH} 4}$ & $S_{\mathrm{NO} 2}$ & $\overline{S_{\mathrm{NO} 3}}$ & $\overline{X_{A}}$ & $X_{N}$ & $X_{I}$ & & \\
\hline \multicolumn{10}{|l|}{ Ammonium oxidizers (A) } \\
\hline 1. Growth & $-\frac{\left(3.43-Y_{A}\right)}{Y_{A}}$ & $-\frac{1}{Y_{A}}-i_{A}$ & $\frac{1}{Y_{A}}$ & - & 1 & - & - & $\mu_{A, \max } \cdot \frac{S_{\mathrm{O} 2}}{K_{A, \mathrm{O} 2}+S_{\mathrm{O} 2}} \cdot \frac{S_{\mathrm{NH} 4}}{K_{\mathrm{NH} 4}+S_{\mathrm{NH} 4}}$ & $X_{A}$ \\
\hline 2. Endogenous respiration & $-\left(1-f_{X I}\right)$ & $i_{A}-i_{I} f_{X I}$ & - & - & -1 & - & $f_{X I}$ & $b_{A} \cdot \frac{S_{\mathrm{O} 2}}{K_{A, \mathrm{O} 2}+S_{\mathrm{O} 2}} \cdot X_{A}$ & \\
\hline 3. Inactivation & - & - & - & - & -1 & - & 1 & $b_{A} \cdot \eta \cdot \frac{S_{\mathrm{O} 2}}{K_{A, \mathrm{O} 2}+S_{\mathrm{O} 2}} \cdot X_{A}$ & \\
\hline \multicolumn{10}{|l|}{ Nitrite oxidizers (N) } \\
\hline 4. Growth & $-\frac{\left(1.14-Y_{N}\right)}{Y_{N}}$ & $-i_{A}$ & $-\frac{1}{Y_{N}}$ & $\frac{1}{Y_{N}}$ & - & 1 & - & $\mu_{N, \max } \cdot \frac{S_{\mathrm{O} 2}}{K_{N, \mathrm{O} 2}+S_{\mathrm{O} 2}} \cdot \frac{S_{\mathrm{NO} 2}}{K_{\mathrm{NO} 2}+S_{\mathrm{NO} 2}}$ & $X_{N}$ \\
\hline 5. Endogenous respiration & $-\left(1-f_{X I}\right)$ & $i_{A}-i_{I} f_{X I}$ & - & - & - & -1 & $f_{X I}$ & $b_{N} \cdot \frac{S_{\mathrm{O} 2}}{K_{N, \mathrm{O} 2}+S_{\mathrm{O} 2}} \cdot X_{N}$ & \\
\hline 6. Inactivation & - & - & - & - & - & -1 & 1 & $b_{N} \cdot \eta \cdot \frac{S_{\mathrm{O} 2}}{K_{N, \mathrm{O} 2}+S_{\mathrm{O} 2}} \cdot X_{N}$ & \\
\hline
\end{tabular}

The biomass components $X_{i}$ are used for both biofilm biomass $f_{i} \rho$ and suspended biomass $u_{i}$. We compute reaction rates $r_{k}(t, z)=\sum_{j=1}^{6} v_{k j} P_{j}\left[\frac{\mathrm{g}}{\mathrm{m}^{3} \cdot \mathrm{d}}\right]$ and growth rates $\mu_{i}(t, z)=\sum_{j=1}^{6} \frac{v_{i j} P_{j}}{f_{i} \rho}[1 / \mathrm{d}]$, see Appendix A 
This biofilm model is a free boundary value problem for four semi-linear diffusionreaction equations describing the substrate concentration profiles across the biofilm $C_{\mathrm{O} 2}(z), C_{\mathrm{NH} 4}(z), C_{\mathrm{NO} 2}(z), C_{\mathrm{NO} 3}(z)$ coupled with two hyperbolic equations with non-local effects for the biomass distribution across the biofilm, $f_{A}(z, t), f_{N}(z, t)$. Note that the equation for $f_{I}(z, t)$ need not be solved due to (13). Although stand alone biofilm models of this type have been used for numerical simulation of wastewater treatment processes for several years (see Wanner et al. 2006), first qualitative mathematical results appeared in the literature only recently (Szomolay et al. 2010; Szomolay 2008) and for microbially simpler systems. These are existence results and results about the stability of steady state solutions. Stability criteria obtained with these techniques, however, are not easy to apply to problems with nonlinear reaction kinetics, since they depend strongly on quantitative aspects of the substrate concentration profiles, for which often only crude estimates are available. Moreover, how to apply these results to study a biofilm model that is embedded in the eight-dimensional reactor mass balance is not obvious. Furthermore, for a much simpler problem, in which only one biomass species and one substrate concentration was considered, and in which case the hyperbolic free-boundary value problem could be reduced to an ordinary differential equation, algebraic complexity prevented a complete analysis of non-trivial equilibria (Mašić and Eberl 2012). Therefore, we cannot expect to be able to obtain much insight into model behavior from analytical techniques, but we have to rely on numerical solutions instead.

Remark 2.2 (Suspended vs. sessile biomass) The mathematical model (1)-(15) does not permit a non-trivial solution without suspended or without sessile biomass. To see this, let us assume that such a solution exists, i.e. a solution with $u_{A}(t)=u_{N}(t)=$ $u_{I}(t)=0$ and with $\lambda(t)>0$ for all $t \in\left(t_{a}, t_{b}\right)$, where $0 \leq t_{a}<t_{b}$. The assertion follows for the suspended biomass fractions by contradiction from (6)-(8). Similarly, if one assumes instead that $\lambda(t)=0$ for all $t \in\left(t_{a}, t_{b}\right)$, but $u_{A}(t)+u_{N}(t)+u_{I}(t)>0$, then a contradiction to (15) is obtained. This simple observation implies that the model (1)-(15) does neither permit a "suspended only" nor a "biofilm only" special solution. However, the mere presence of both types of biomass does not imply that they both contribute at similar levels to the nitrification process. To investigate this question will be one of the main thrusts of the numerical simulations documented below.

Remark 2.3 (Equilibria) A rigorous mathematical analysis of the steady states of the model and their stability is difficult. In Appendix B, we discuss this and compare the numerical findings for the multi-species model with the analytical results of the simpler single-species model of Mašić and Eberl (2012).

\section{Model Parameters and Numerical Simulations}

\subsection{Model Parameters}

In many wastewater treatment reactors, oxygen is externally supplied to avoid that it becomes the process limiting substrate (Rittmann and McCarty 2001). To account for 
Table 2 Reactor dimensions

\begin{tabular}{llll}
\hline Parameter & Value & Unit & Reference \\
\hline Radius & 0.08 & $\mathrm{~m}$ & assumed \\
Height & 0.3 & $\mathrm{~m}$ & assumed \\
Inner surface area $A_{\text {reactor }}$ & 0.17 & $\mathrm{~m}^{2}$ & calculated from radius and height \\
Volume $V$ & 0.006 & $\mathrm{~m}^{3}$ & calculated from radius and height \\
Suspended carrier area & 0.0068 & $\mathrm{~m}^{2}$ & Mašić et al. (2010) \\
\hline
\end{tabular}

this, the oxygen bulk concentration is kept at a constant value, and the corresponding reactor mass balance in (1) is removed from the system. However, across the biofilm, oxygen is consumed by the bacteria. Therefore, the diffusion-reaction equation (4) still needs to be considered for oxygen. Furthermore, the volume fraction for inert biomass in the biofilm can be obtained with Eq. (13), wherefore the corresponding equation in (11) need not be solved. The remaining nine equations (1), (6)-(8), (11), (15) are coupled with four steady state diffusion-reaction equations (4) for the dissolved substances in the biofilm, which need to be solved in every time step of the dynamic model. This model was implemented for simulation using MATLAB R2010b, attaining moderate computational complexity. The diffusion-reaction equations (4) were solved with the built-in solver bvp $4 \mathrm{c}$, a finite difference code using the threestage Lobatto IIIa formula, which is a collocation formula (The Mathworks 2012). Most of the other equations were solved using in-house finite difference schemes. Integrals were approximated with the trapezoidal rule except the integrals in (11) and (15) where we used the built-in function quadl, a high order method using an adaptive Gauss/Lobatto quadrature rule (The Mathworks 2012). Profiling of the simulation code showed that most computation time was spent on solving the boundary value problem (4), which is why most researchers use an approximation of the problem. The time to compute the steady-state values of the system with desired accuracy by time-stepping the thirteen equations to steady state were in the order of hours to days, depending on the specific combination of parameters. The simulations were run on a Dell Precision T5500 workstation with two quadcore Intel Xeon X5550 processors and 12 GB RAM and on a HP Compaq nc6320 Business Notebook with an Intel Core 2 Duo T7200 processor and 4 GB RAM.

The biofilm reactor in our simulations is a cylindrical reactor with an open top, a volume $V=0.006 \mathrm{~m}^{3}$ and an inner surface area $A_{\text {reactor }}=0.17 \mathrm{~m}^{2}$; see Table 2 . The additional suspended carriers that can be added to the system each have an area of $0.0068 \mathrm{~m}^{2}$. With a maximum amount of 150 carriers, this results in a total available area for biofilm colonization $A$ between $0.17 \mathrm{~m}^{2}$ and $1.19 \mathrm{~m}^{2}$. Note that $A$ (area) and $c$ (number of suspended carriers) will be used interchangeably throughout the paper.

The reaction parameters that we used for our study have been collected from the literature and were validated against experimental data in Mašić et al. (2010). These experiments were carried out at $10{ }^{\circ} \mathrm{C}$, to account for water temperatures in colder climates, such as Scandinavia or the Canadian Spring and Fall. At these low temperatures, the growth rates for NOB are higher than for AOB, contrary to what is 
Table 3 Model parameters at $10^{\circ} \mathrm{C}$

\begin{tabular}{|c|c|c|c|c|}
\hline Symbol & Name & Value & Unit & Reference \\
\hline$\alpha$ & attachment rate & 1 & $d^{-1}$ & assumed \\
\hline$b_{A}$ & decay rate of $\mathrm{AOB}$ & 0.04 & $d^{-1}$ & Salem et al. (2006) \\
\hline$b_{N}$ & decay rate of $\mathrm{NOB}$ & 0.08 & $d^{-1}$ & Salem et al. (2006) \\
\hline$D$ & dilution rate & varied & $\mathrm{d}^{-1}$ & - \\
\hline$D_{\mathrm{O} 2}$ & diffusion coefficient for $\mathrm{O}_{2}$ & $9.93 \cdot 10^{-5}$ & $\mathrm{~m}^{2} \mathrm{~d}^{-1}$ & Wik (1999) \\
\hline$D_{\mathrm{NH} 4}$ & diffusion coefficient for $\mathrm{NH}_{4}^{+}$ & $9.13 \cdot 10^{-5}$ & $\mathrm{~m}^{2} \mathrm{~d}^{-1}$ & Wik (1999) \\
\hline$D_{\mathrm{NO} 2}$ & diffusion coefficient for $\mathrm{NO}_{2}^{-}$ & $7.93 \cdot 10^{-5}$ & $m^{2} d^{-1}$ & Wik (1999) \\
\hline$D_{\mathrm{NO} 3}$ & diffusion coefficient for $\mathrm{NO}_{3}^{-}$ & $7.93 \cdot 10^{-5}$ & $\mathrm{~m}^{2} \mathrm{~d}^{-1}$ & Wik (1999) \\
\hline$E$ & erosion parameter & 1000 & $\mathrm{~m}^{-1} \mathrm{~d}^{-1}$ & assumed \\
\hline$\eta$ & anoxic reduction factor for $b_{A}$ or $b_{N}$ & 0.5 & - & Elenter et al. (2007) \\
\hline$f_{X I}$ & $\begin{array}{l}\text { fraction of inert biomass produced } \\
\text { by endogenous respiration }\end{array}$ & 0.1 & - & Picioreanu et al. (2004) \\
\hline$i_{A}$ & nitrogen content of active biomass & 0.07 & - & Picioreanu et al. (2004) \\
\hline$i_{I}$ & nitrogen content of inactive biomass & 0.02 & - & Picioreanu et al. (2004) \\
\hline$K_{A, \mathrm{O} 2}$ & half-saturation Monod coeff. for $\mathrm{AOB}, \mathrm{O}_{2}$ & 0.5 & $\mathrm{gm}^{-3}$ & Pai (2007) \\
\hline$K_{N, \mathrm{O} 2}$ & half-saturation Monod coeff. for $\mathrm{NOB}, \mathrm{O}_{2}$ & 0.5 & $\mathrm{gm}^{-3}$ & Pai (2007) \\
\hline$K_{\mathrm{NH} 4}$ & half-saturation Monod coeff. for $\mathrm{AOB}, \mathrm{NH}_{4}^{+}$ & 0.1690 & $\mathrm{gm}^{-3}$ & Mašić et al. (2010) \\
\hline$K_{\mathrm{NO} 2}$ & half-saturation Monod coeff. for $\mathrm{NOB}, \mathrm{NO}_{2}^{-}$ & 0.302 & $\mathrm{gm}^{-3}$ & Wiesmann (1994) \\
\hline$\mu_{A, \max }$ & maximum specific growth rate for $\mathrm{AOB}$ & 0.3082 & $d^{-1}$ & Wyffels et al. (2004) \\
\hline$\mu_{N, \max }$ & maximum specific growth rate for NOB & 0.4015 & $d^{-1}$ & Wyffels et al. (2004) \\
\hline$\rho$ & biomass density & 10000 & $\mathrm{gm}^{-3}$ & Picioreanu et al. (2004) \\
\hline$Q$ & flow through the reactor & varied & $\mathrm{m}^{3} \mathrm{~d}^{-1}$ & - \\
\hline$Y_{A}$ & biomass yield coefficient for $\mathrm{AOB}$ & 0.15 & - & Wyffels et al. (2004) \\
\hline$Y_{N}$ & biomass yield coefficient for NOB & 0.041 & - & Wyffels et al. (2004) \\
\hline
\end{tabular}

commonly found in warmer temperatures (Hunik et al. 1994; Hellinga et al. 1998). The reaction parameters are summarized in Table 3.

We have chosen the same stoichiometry and kinetic parameters for suspended biomass as for biofilm biomass, due to the poor understanding and availability of reliable parameters (Boltz et al. 2009a). Being aware that this choice limits our numerical study, we have reduced the complexity from 42 parameters to 28 .

All simulations, unless otherwise stated, start with an initial biofilm thickness and suspended biomass

$$
\lambda(0)=10 \mu \mathrm{m}, \quad u_{A}(0)=u_{N}(0)=20 \mu \mathrm{g},
$$

i.e. we assume an equal amount of AOB and NOB and no inerts initially. Thus,

$$
f_{A}(z, 0)=f_{N}(z, 0)=0.5, \quad f_{I}(z, 0)=0 .
$$

The initial concentrations of the dissolved compounds are

$$
S_{\mathrm{NH} 4}(0)=30 \mathrm{~g} / \mathrm{m}^{3}, \quad S_{\mathrm{NO} 2}(0)=0 \mathrm{~g} / \mathrm{m}^{3}, \quad S_{\mathrm{NO} 3}(0)=1 \mathrm{~g} / \mathrm{m}^{3}
$$


and dissolved oxygen $S_{\mathrm{O} 2}$ is kept constant at $5 \mathrm{~g} / \mathrm{m}^{3}$. The simulations are terminated once they attain steady state in all variables, i.e. when the changes in the state vector between two consecutive time steps are small.

\subsection{Simulation Experiments}

In the numerical simulations we used the hybrid model, defined by Eqs. (1), (6)(8), (11), (15), and (4), to investigate the contribution of suspended biomass to the overall reactor performance. Primarily two parameters have been varied during the simulations: the dilution rate $D$ between $0 \mathrm{~d}^{-1}$ and $160 \mathrm{~d}^{-1}$, and the number of suspended carriers that supply additional colonizable area for the biofilms between 0 and 150 with an increment of 25 carriers resulting in a total of 133 simulations for the hybrid model.

For comparison purposes, we also carried out simulations in which we only considered biofilm bound biomass, but no suspendeds and vice versa. We used the same numerical code as previously compiled for the hybrid model, but set the attachment rate to zero when appropriate, and set the initial data accordingly. Furthermore, in the suspendeds-only case, we did not solve the equations for diffusion-reaction (4), biofilm volume fractions (11) and biofilm thickness (15), and $D$ was varied on a grid of 48 points between $0 \mathrm{~d}^{-1}$ and $1 \mathrm{~d}^{-1}$. For biofilm-only, we did not solve the suspended biomass mass balances (6)-(8), carrying out 133 simulations corresponding to the simulation of the hybrid model. Note that the solution to the biofilm-only model is not a special solution of the hybrid model as per Remark 2.2.

To study the effects of changes in attachment and detachment rates for various reactor conditions, additional simulations were performed where we simultaneously varied the dilution rate $D$ (between 0.5 and $10 \mathrm{~d}^{-1}$ ), the attachment rate $\alpha$ (between 0.1 and $1.5 \mathrm{~d}^{-1}$ ), the number of suspended carriers $c$ (between 0 and 150) and the erosion parameter $E$ (between 100 and $1500 \mathrm{~m}^{-1} \mathrm{~d}^{-1}$ ), resulting in 256 additional simulations. For comparison purposes, also 64 corresponding simulations were carried out for the biofilm-only model, where the attachment rate does not need to be considered.

\section{Results and Discussion}

In this section, we summarize and discuss the results of simulations of the model under varying reactor operation conditions, in terms of dilution rate $D$ and colonizable surface area $A$, and for varying detachment rates that are correlated with reactor hydraulic load. In all simulations, steady state was attained eventually. The primary focus of our computational analysis will be on reactor performance and microbial ecology at steady state and the comparison of the hybrid model that considers biofilm and suspended biomass with the biofilm-only model.

\subsection{Detailed Documentation of a Typical Simulation of the Hybrid Model}

A typical simulation of our model is presented in Figs. 1 and 2. The dilution rate in this simulation is set to $D=5 /$ day, and the number of biofilm carriers in the reactor is $c=50$. 

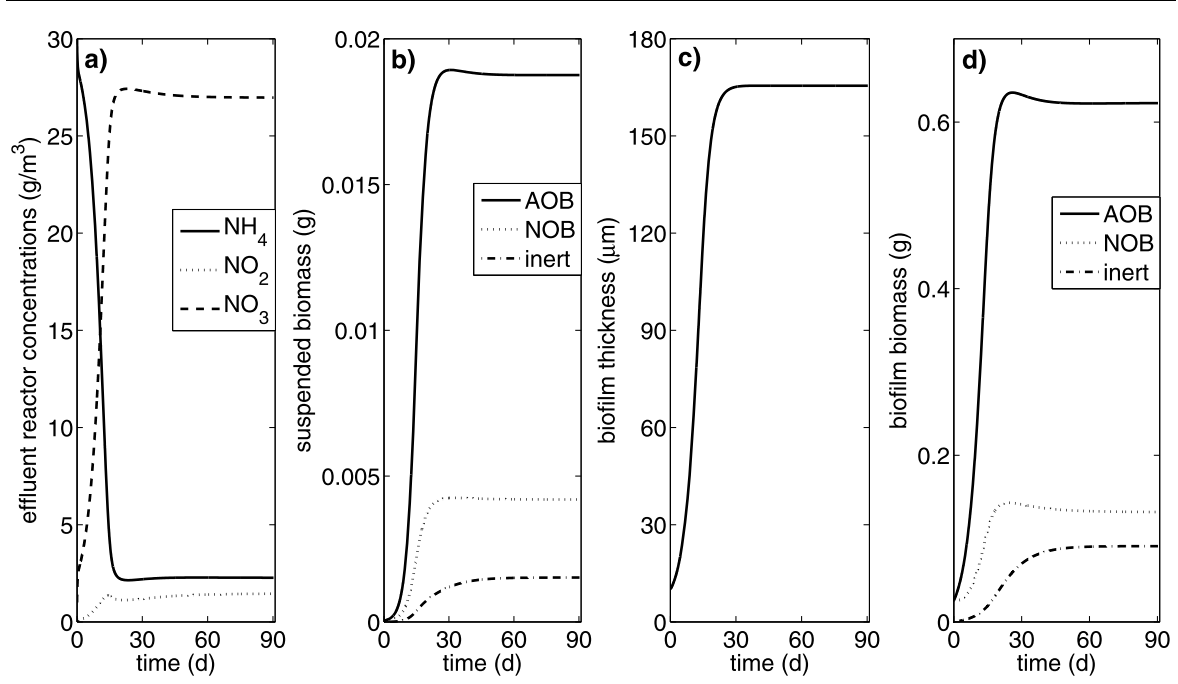

Fig. 1 Typical time-dependent simulations of (a) effluent concentrations of ammonium (solid), nitrite (dot), and nitrate (dash); (b) amount of AOB (solid), NOB (dot), and inerts (dash-dot) in suspended form; (c) biofilm thickness; (d) amount of AOB (solid), NOB (dot), and inerts (dash-dot) in biofilm form; in the hybrid model at $D=5 /$ day and $c=50$
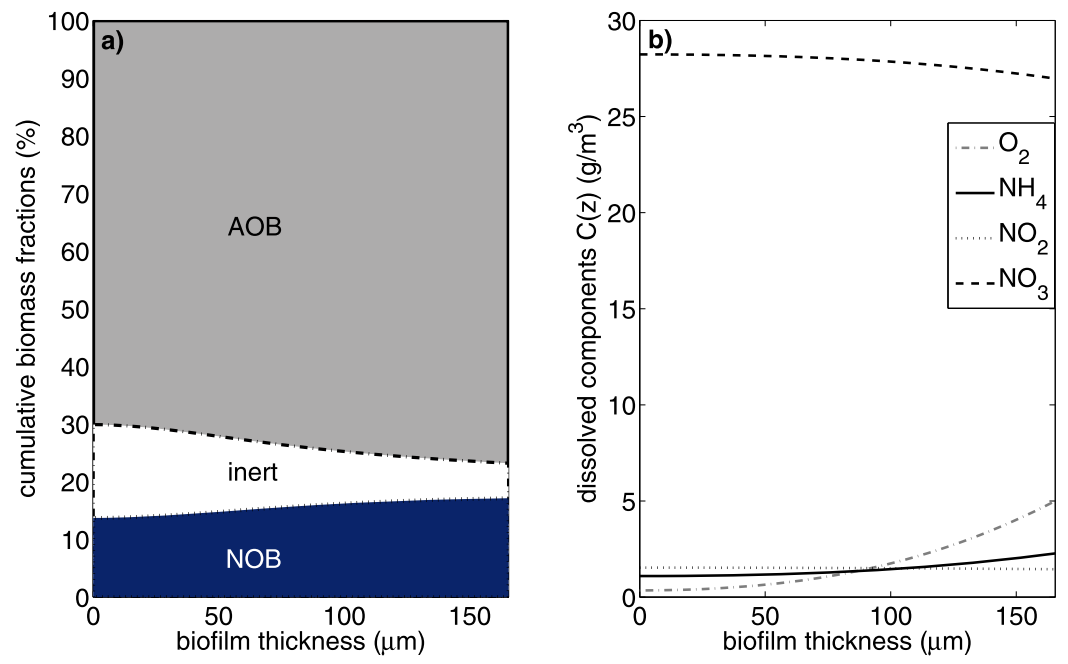

Fig. 2 Spatial distribution across the biofilm at steady state for $D=5 /$ day and $c=50$ : (a) biomass fractions AOB (gray), NOB (dark blue) and inerts (white) in the biofilm; (b) concentrations $C(z)$ of oxygen (dash-dot gray), ammonium (solid), nitrite (dot) and nitrate (dash) (Color figure online)

The time evolution of the system is documented in Fig. 1. The ammonium concentration in the effluent starts at $S_{\mathrm{NH} 4}(0)=30 \mathrm{~g} / \mathrm{m}^{3}$ and drops quickly down to a much lower value due to consumption by AOB, leveling off after approximately 25 days. Nitrite, the outcome of AOB consumption, is initially absent from the system, 
but is produced and reaches a plateau value, albeit a small one. Most of the nitrite is converted to nitrate by NOB, which is why the nitrate concentration increases rapidly from $S_{\mathrm{NO} 3}(0)=1 \mathrm{~g} / \mathrm{m}^{3}$ and levels off at the same time as $S_{\mathrm{NH} 4}$.

The transient behavior of the substrates is reflected in the transient behavior of suspended (Fig. 1b) and sessile biomass fractions (Figs. 1c, d) in the system. Suspended AOB increases to eventually level off, while the faster growing NOB level off at a smaller value. AOB are initially exposed to abundant nutrient and oxygen and are able to expand their biomass to a greater extent than NOB, which have to wait for the nitrite to be produced. Suspended inerts also accumulate in the reactor and reach a plateau, however, at a later time than the active biomass fractions. The biofilm in the system attains a thickness of $\lambda=165 \mu \mathrm{m}$ (see Fig. 1c) at about the same time that the suspended biomass fractions attain their equilibrium values. In the biofilm, a similar development is occurring as in the suspended phase due to nutrient and oxygen availability. However, NOB attains a higher proportion of active biomass relative to AOB, compared to the suspended populations. Inerts in the biofilm eventually level off at a higher ratio to active biomass than for the suspended bacteria. This is likely due to substrate limitations in the inner layers of the biofilm. Overall, the amount of biomass in the biofilm is by more than one order of magnitude larger than in the suspended mode of growth.

The spatial distribution of biomass and substrates across the biofilm at steady state is documented in Figs. 2a, b. AOB is the clearly dominating species, as already indicated in Fig. 1d. Both active biomass fractions AOB and NOB occupy more volume in the outer layer of the biofilm than in the inner layer of the biofilm. Inerts, on the other hand, occupy more volume in the inner layers than in the outer layers. This is explained by the spatial distributions of the growth limiting substrates.

Oxygen, which is kept at a constant value in the liquid phase by aeration, and consumed for growth and endogenous respiration of both species, decreases in concentration from the biofilm/water interface to the substratum. At the biofilm/water interface the bulk oxygen concentration is one order of magnitude higher than the half saturation concentrations $K_{A, \mathrm{O} 2}$ and $K_{N, \mathrm{O} 2}$, whereas in the inner layers of the biofilm, the oxygen concentration attains approximately the same order of magnitude as the half saturation concentrations, indicating oxygen limitation of growth there. Lack of oxygen in the inner layers of the biofilm explains the accumulation of inert biomass at the substratum. The concentration of ammonium $S_{\mathrm{NH} 4}$, which is continuously supplied to the reactor externally, is higher at the biofilm water interface than in the inner layers. Nevertheless, the lowest concentration value at the substratum is still one order of magnitude higher than the half saturation concentration $K_{\mathrm{NH} 4}$, which indicates that ammonium does not limit growth in this simulation, due to the high external supply, i.e. more ammonium is supplied than the biomass is able to consume. The concentration of nitrite $S_{\mathrm{NO} 2}$, which is not externally supplied to the reactor, remains approximately constant across the biofilm, indicating an equilibrium between the liquid and biofilm phase. Also, this concentration value is substantially higher than the half-saturation concentration $K_{\mathrm{NO} 2}$. The product nitrate $S_{\mathrm{NO} 3}$, which is continuously supplied to the reactor in a small amount, attains its highest values in the inner layers, indicating a substrate flux from the biofilm into the liquid phase, due to the strong activity in the biofilm. 
4.2 For Given Reaction Parameters, how Do the Operating Conditions of the Reactor, Expressed in Terms of Hydraulic Load, $D$ and Colonizable Surface Area A, Affect the Biofilm Nitrification Process Qualitatively and Quantitatively?

Figure 3 is included here for the reader's convenience, with a detailed description and further results found online as supplementary material to this article. We plot the steady state values of ammonium, nitrite, and nitrate concentration, ammonium removal, production of nitrite and nitrate and amount of AOB, NOB, and inert biomass in the biofilm, in the hybrid model and in the biofilm-model for various amounts of suspended carriers $c$ and various dilution rates $D$.

The dilution rate controls the amount of substrate that is supplied to the reactor as well as the removal of suspended biomass. Colonizable surface area indirectly governs the amount of biofilm in the reactor. The dilution rates were varied between 0 and $160 \mathrm{~d}^{-1}$ and the number of suspended carriers between 0 and 150 carriers. The combined effect of variation in these operating conditions shows that changes in dilution rate and colonizable surface area have a great impact on the performance of the hybrid reactor at steady state (see Fig. 3). Detailed results can be found in the online supplementary material to this article.

Complete conversion of ammonium to nitrate is obtained in the simulations for all surface areas at very small dilution rates (see Figs. 3a, c), at which only a small amount of substrate enters the reactor. When the dilution rate is increased, we see a domination of ammonium over nitrate in the effluent at small surface areas and vice versa at large $A$. Too small surface areas carry an insufficient amount of biofilm that is unable to consume all the incoming ammonium, resulting in a high concentration of unused ammonium leaving the reactor with the effluent. When the area is increased enough, biofilm can be established to convert the ammonium to high levels of nitrate. Effluent nitrite concentration increases when dilution rate and surface area are increased, with high peaks of nitrite for small $D$ and $A$; see Fig. 3b. Removal of ammonium and production of nitrate in Figs. $3 \mathrm{~d}$ and $\mathrm{f}$ increase as $D$ and $A$ increase, with ammonium removal reaching a plateau for large $D$ while nitrate production eventually begins to decrease. The decrease in nitrate production is connected to the increase in net production of nitrite, which generally increases as $D$ increases and as $A$ decreases; see Fig. 3e. Very large amounts of ammonium, which are supplied by large dilution rates, cause an increase in $\mathrm{AOB}$ and a higher demand for oxygen. The remaining oxygen that is available for NOB growth is insufficient to maintain a large amount of biomass, i.e. nitrate production decreases.

Increased surface area allows for more biofilm growth; however, the nutrients can be growth limiting rather than the surface area. Although the overall biofilm biomass increases as surface area is increased, the biofilm thickness itself decreases. On the other hand, increased dilution rate supplies more substrates to the reactor, causing an increased biomass growth. Biomass composition within the biofilm displays an increase in all biomass types as surface area is increased, see Fig. 4a. However, an increased dilution rate causes the ammonium oxidizers to increase due to higher substrate supply and to eventually reach a plateau. It causes the nitrite oxidizers to reach a maximum and subsequently to decrease, and the inert biomass to decrease in general. 

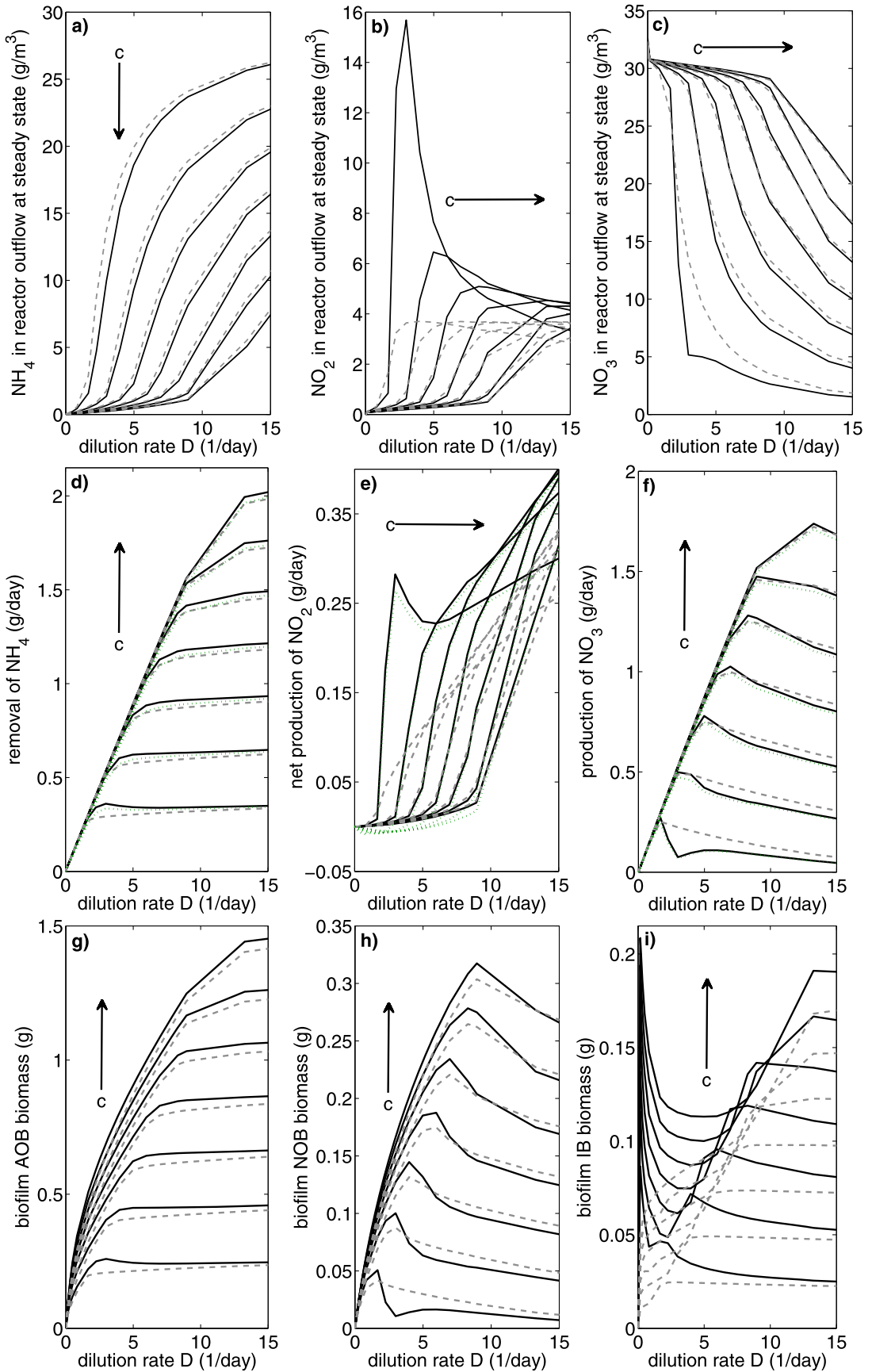

Fig. 3 Steady state results for varying dilution rates $D$ and amount of suspended carriers $c$ : Concentration $\left[\mathrm{g} / \mathrm{m}^{3}\right]$ of (a) ammonium, (b) nitrite, (c) nitrate in reactor outflow; Removal $[\mathrm{g} / \mathrm{d}]$ of (d) ammonium; Production $[\mathrm{g} / \mathrm{d}]$ of $(\mathbf{e})$ nitrite, (f) nitrate; Amount $[\mathrm{g}]$ of biomass type (g) AOB, (h) NOB, (i) inert; in the hybrid model (solid), in the biofilm model (dashed) and the biofilm contribution in the hybrid model (dotted, only in (d)-(f)) (Color figure online) 

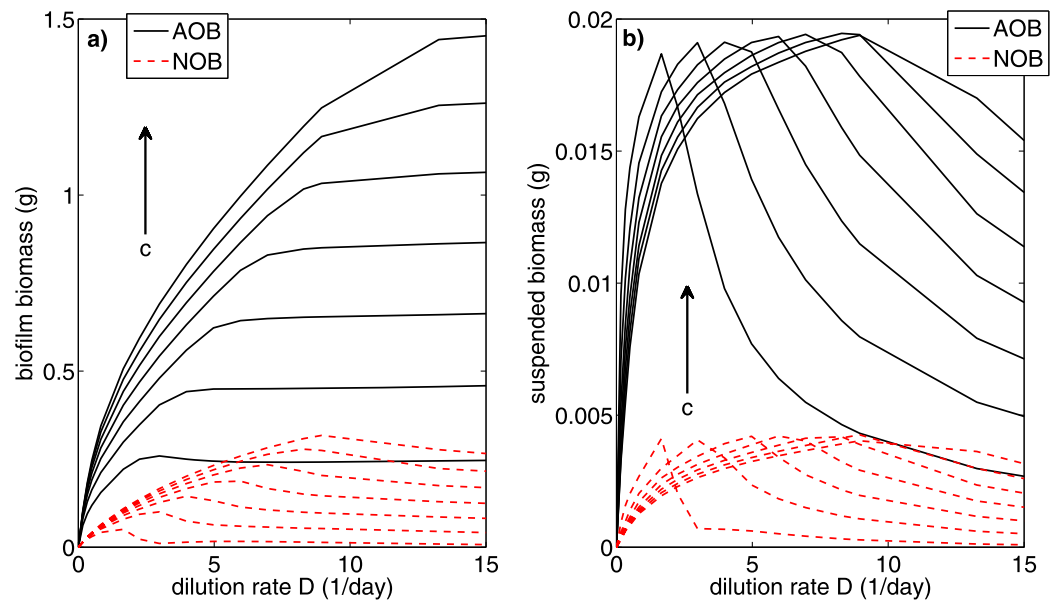

Fig. 4 Steady state values for varying dilution rates $D$ and amount of suspended carriers $c$ : AOB (solid) and NOB (dashed) biomass [g] in (a) biofilm, (b) suspended form, in the hybrid model (Color figure online)

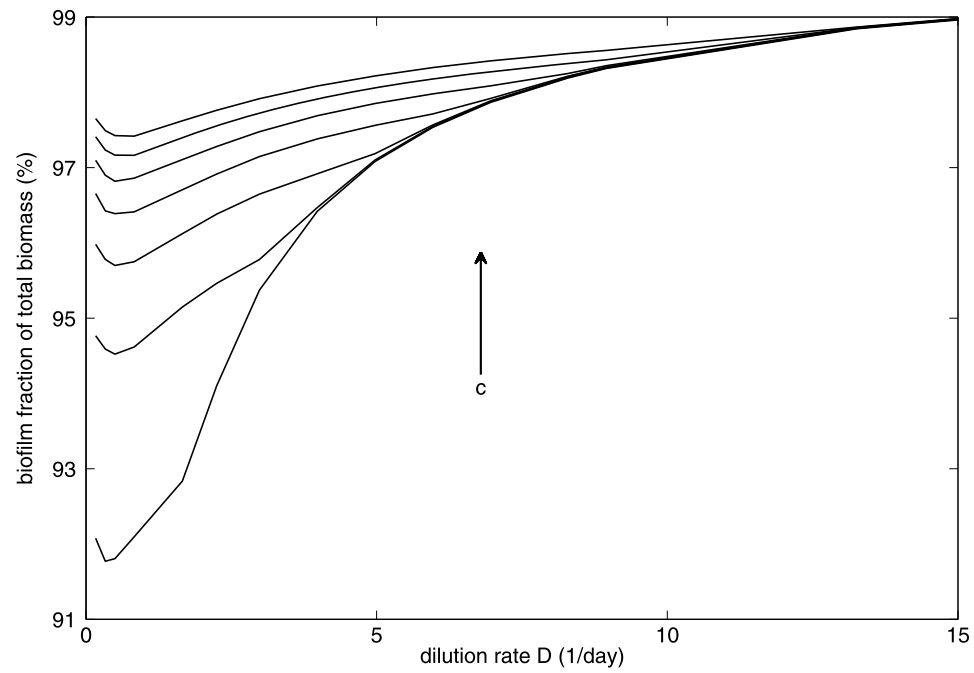

Fig. 5 Steady state values for varying dilution rates $D$ and amount of suspended carriers $c$ : Percentage of biofilm biomass in the total amount of biomass in the hybrid model (solid)

The decrease in NOB biomass occurs due to higher demand by AOB for oxygen, for which the two species compete. AOB and NOB in suspended biomass reach maxima as $D$ is increased (see Fig. $4 \mathrm{~b}$ ) after which they decrease, i.e. lose biomass due to washout from the reactor. For small dilution rates the suspended biomass decreases as $A$ is increased, with the opposite occurring for large dilution rates.

Throughout the simulations, the hybrid model is dominated by the biofilm biomass compared to suspended biomass; see Fig. 5. The amount of biofilm is increased as $D$ 
and $A$ are increased, largely due to washout of suspended biomass and an increased biofilm area, respectively. Suspended biomass accounts for at most $8 \%$ of the total biomass in the reactor, for small dilution rate, and colonizable surface area.

4.3 What Is the Quantitative Contribution of the Suspended Bacteria to the Predominantly Biofilm Controlled Nitrification Process? Can a Simpler Model that Considers Only the Biofilm but Does Not Explicitly Account for Transformation Processes in the Suspended Phase Give a Sufficiently Good Approximation of the Process?

Depending on the reactor operating conditions, suspended biomass can account for up to $8 \%$ of the total biomass in the system. For higher dilution rates, this number might be as small as $1 \%$ due to increased washout. This statement holds similarly for AOB and NOB; see Figs. 3g, h. More AOB biomass is found in the biofilm in the hybrid model than in the biofilm-only model, which is partly reversed for the NOB biomass for large dilution rates. The explanation lies in a combined effect of biomass attachment and competition for oxygen. In the hybrid model, biomass is continuously attached from the suspended phase to the biofilm, thereby keeping more biomass in the system. AOB dominates in the suspended phase at large dilution rates, while NOB is largely washed out. Therefore, more AOB is attached to the biofilm in the hybrid model. The increased amount of AOB consumes more oxygen, leaving less oxygen available for NOB growth, which is why the amount of NOB in the biofilm eventually decreases.

The second step of nitrification, i.e. conversion of nitrite to nitrate, is significantly affected by the presence of suspended biomass in the reactor. More detailed results are found in the online supplementary material to this article. The effluent nitrite concentration in Fig. $3 \mathrm{~b}$ and its net production rate in Fig. 3e differ greatly between the biofilm model and the hybrid model, in particular for small colonizable surface areas, where competition for oxygen between biofilm and suspended biomass and oxygen limitation cause nitrite accumulation, which is not desired in nitrification. The amount of NOB in Fig. $3 \mathrm{~h}$ is only slightly affected by the presence of suspended biomass, whereas we observe significant effects on inert biomass in Fig. 3i. Not only does a larger quantity of inerts exist in the hybrid model, they also respond stronger to variations in dilution rate and surface area. Consequently, we have a thicker and less active biofilm in the hybrid model.

Overall removal of ammonium and production of nitrate in the reactor (Figs. 3d, f) are insignificantly affected by the presence of suspended biomass in the reactor, even as dilution rate and colonizable surface area are varied. The effluent concentrations of ammonium and nitrate along with the amount of AOB in the reactor (Figs. 3a, c, g) are likewise unaffected by the suspended biomass. The overall behavior of a biofilm model and a hybrid model is indistinguishable when focusing on the aforementioned removal rates and concentrations.

Despite a quantitative domination by the biofilm, we find detectable effects on the system by the suspended biomass, whose survival in the reactor is prolonged through the biomass exchange between attached and unattached form. When studying a model containing only suspended biomass, we observe monumental differences compared 
to the biofilm and hybrid models, see figures in the supplementary material. In a suspended-only model, biomass is very strongly affected by the flow rate, reaching washout of biomass for dilution rates of the same order of magnitude as the biomass growth rates. Washout of suspended biomass in the hybrid model is noticeable for higher dilution rates, although never completely achieved.

\subsection{What Are the Effects of Attachment and Detachment in the Hybrid Model?}

Re-attachment of suspended biomass and detachment of biofilm biomass are complex, not well understood processes that are approximated by relatively simple expressions. The detachment model that we use here is fairly common in biofilm modeling and assumes that the detachment rate is proportional to the biofilm thickness. Attachment is modeled using a constant rate. We conduct a sensitivity analysis, whereby we vary $\alpha$ and $E$ by more than one order of magnitude, to assess the role of these mass exchange processes for the overall reactor system. In Sect. 4.2, we have seen that the dilution rate and the colonizable surface area have a strong effect on the process parameters and that in particular biofilm composition can vary greatly in dependence of these parameters. Therefore, in order to obtain a comprehensive picture of parameter interdependencies, identifying a representative set of reactor operating conditions is not possible for the sensitivity analysis with respect to attachment and detachment, but we vary $\alpha$ and $E$ together with the reactor conditions $D$ and $c$. We vary $E$ across $100,500,1000$, and $1500 / \mathrm{md}$, the $\alpha$ across $0.1,0.5,1.0$, and $1.5 / \mathrm{d}$, the $D$ across $0.5,2.5,5.0$, and $10 / \mathrm{d}$, and the number of suspended carriers $c$ across 0,50 , 100 , and 150 , resulting in 256 parameter combinations.

Regarding their sensitivity with respect to $\alpha, c, D, E$, the dependent variables and other quantities of interest in the model can be grouped by processes, which are roughly connected to the first and second steps of nitrification. In Fig. 6, we show the steady state values of three representatives of these groups. Each sub-figure in Fig. 6 contains 16 curves of different line style and color, representing the different combinations of $D$ and $\alpha$. The variables in the first two rows of Fig. 6, overall ammonium removal and biofilm $\mathrm{AOB}$ biomass, represent the first step, along with ammonium concentration in the reactor and suspended AOB biomass (data not shown). NOB biofilm biomass in the third row represents the second group, to which also nitrite net production and concentration as well as suspended NOB biomass belong (data not shown).

We observe that the curves in many sub-figures are grouped by line style, indicating that changes in $\alpha$ have a small effect on the steady state outcome of the model. This is particularly visible in the top right sub-figure of ammonium removal at $c=150$, where all the curves of one line style are located on top of each other, indistinguishable by color. On the other hand, $\alpha$ has a more pronounced effect on biofilm NOB biomass at $c=0$ in the bottom left subfigure. A higher $\alpha$ retains more biomass in the reactor through an increased attachment to the biofilm, thereby avoiding a risk of washout. This effect is visible in the AOB sub-figures, where the curves for each line style are ordered such that the highest $\alpha$ attains the largest amount of biomass.

The curves in Fig. 6 are all shown as functions of the erosion parameter $E$. A higher $E$ causes a higher detachment of biomass from the biofilm, i.e. a thinner 

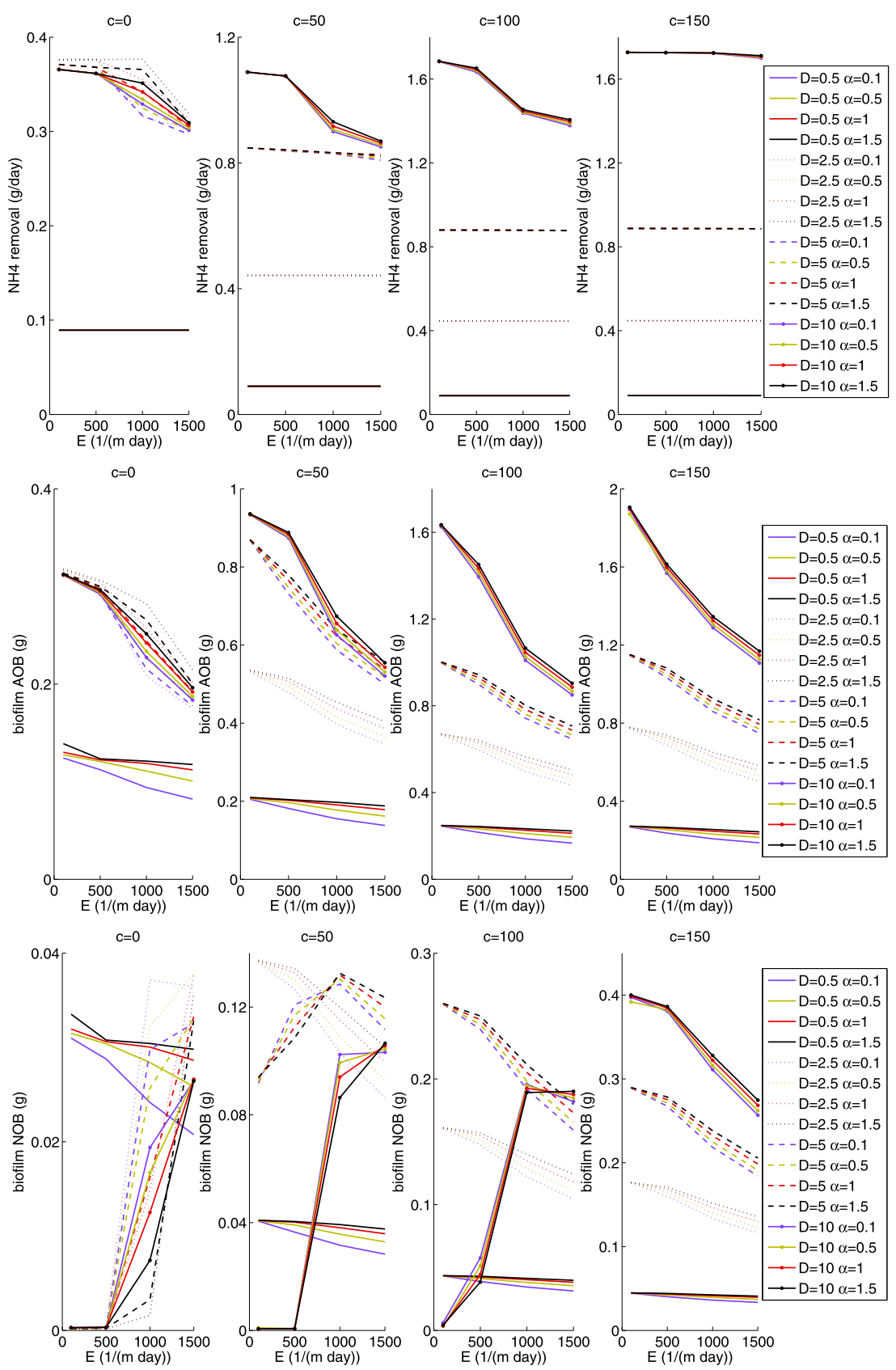

Fig. 6 Steady state values of ammonium removal $\left[\mathrm{g} / \mathrm{m}^{3}\right]$ (1st row), biofilm AOB $[\mathrm{g}]$ (2nd row) and biofilm NOB [g] (3rd row) in the hybrid model with $c=0,50,100,150$ suspended carriers (1st-4th column) as functions of the erosion parameter $E$. Variation in dilution rates $D\left[\mathrm{~d}^{-1}\right]$ represented by line style: $D=0.5$ (solid), $D=2.5$ (dotted), $D=5$ (dashed), $D=10$ (solid w. full circles); variation in attachment rates $\alpha$ $\left[\mathrm{d}^{-1}\right.$ ] represented by color: $\alpha=0.1$ (purple), $\alpha=0.5$ (yellow), $\alpha=1$ (red), $\alpha=1.5$ (black) (Color figure online) 
biofilm. Such an effect is clearly visible in the AOB sub-figures, with a more pronounced effect for larger $c$ and higher $D$, at which more nutrients are supplied to the reactor, resulting in a thicker biofilm on which the effect of increased detachment is noticeable. The behavior of the NOB biomass at $c=0,50$, and 100 shows steeply increasing functions for the higher dilution rates. Large amounts of freshly supplied ammonium at high dilution rates result in excess growth of AOB biomass. Since the NOB biomass is involved in the second step of nitrification, almost all oxygen is consumed by AOB in the first step, leaving no oxygen for the aerobic growth reactions of NOB, which are thus out-competed. This type of behavior is more evident in thicker biofilms that have a large amount of inert biomass due to oxygen depletion. Through an increase of detachment, the biofilm thickness decreases by losing the outer active layer, enabling oxygen to penetrate the previously deeper parts of the biofilm, thus allowing for more NOB growth. Ammonium removal is largely unaffected by erosion, with most curves in the first row in Fig. 6 showing up as constant lines. The AOB biomass in the reactor is able to consume equal amounts of ammonium regardless of the $\alpha$ and $E$ values, indicating that oxygen is the limiting factor in the reaction.

Our results indicate that the erosion, as expressed by $E$, particularly affects the steady state values of the intermediate compounds and processes in the model, such as the nitrite concentration and the NOB biofilm biomass. On the other hand, the re-attachment description, expressed in terms of $\alpha$, shows no such pronounced and consistent effect.

\subsection{How Do $\alpha, D, c$, and $E$ Affect the Relative Differences Between the Hybrid and the Biofilm-Only Model?}

Re-attachment of suspended biomass is not considered in the simpler biofilm-only model. Varying $E, D, c$ over the same ranges as in the previous section, we conduct additional $4^{3}=64$ simulations of the simpler model. We compare the biofilm-only against the hybrid model by analyzing the differences in the quantities of interest: ammonium removal, production of nitrite and nitrate, nitrite concentration, biofilm thickness, biofilm AOB, NOB, and inert biomass. For a combination of $c, D, E$, we compute the relative differences $\delta_{j}(c, D, E)$ between the models

$$
\delta_{j}(c, D, E)=\frac{\left|\bar{q}_{j, h}(c, D, E)-q_{j, b}(c, D, E)\right|}{\bar{q}_{j, h}(c, D, E)},
$$

where $q_{j, b}(c, D, E)$ denotes the quantity in the biofilm model. The averaged quantity $\bar{q}_{j, h}(c, D, E)$ in the hybrid model with respect to $\alpha$ is obtained by

$$
\bar{q}_{j, h}(c, D, E)=\frac{1}{n_{\alpha}} \sum_{r=1}^{n_{\alpha}} q_{j, h}\left(c, D, E, \alpha_{r}\right)
$$

which is substituted directly into (19).

These data are plotted in Fig. 7. For every pair $E, D$, we represent for every $c$ the relative differences between model predictions for the quantities of interest as circles with radius $\delta_{j}$. These circles are color coded with $c=0$ black, $c=50 \mathrm{red}, c=100$ 

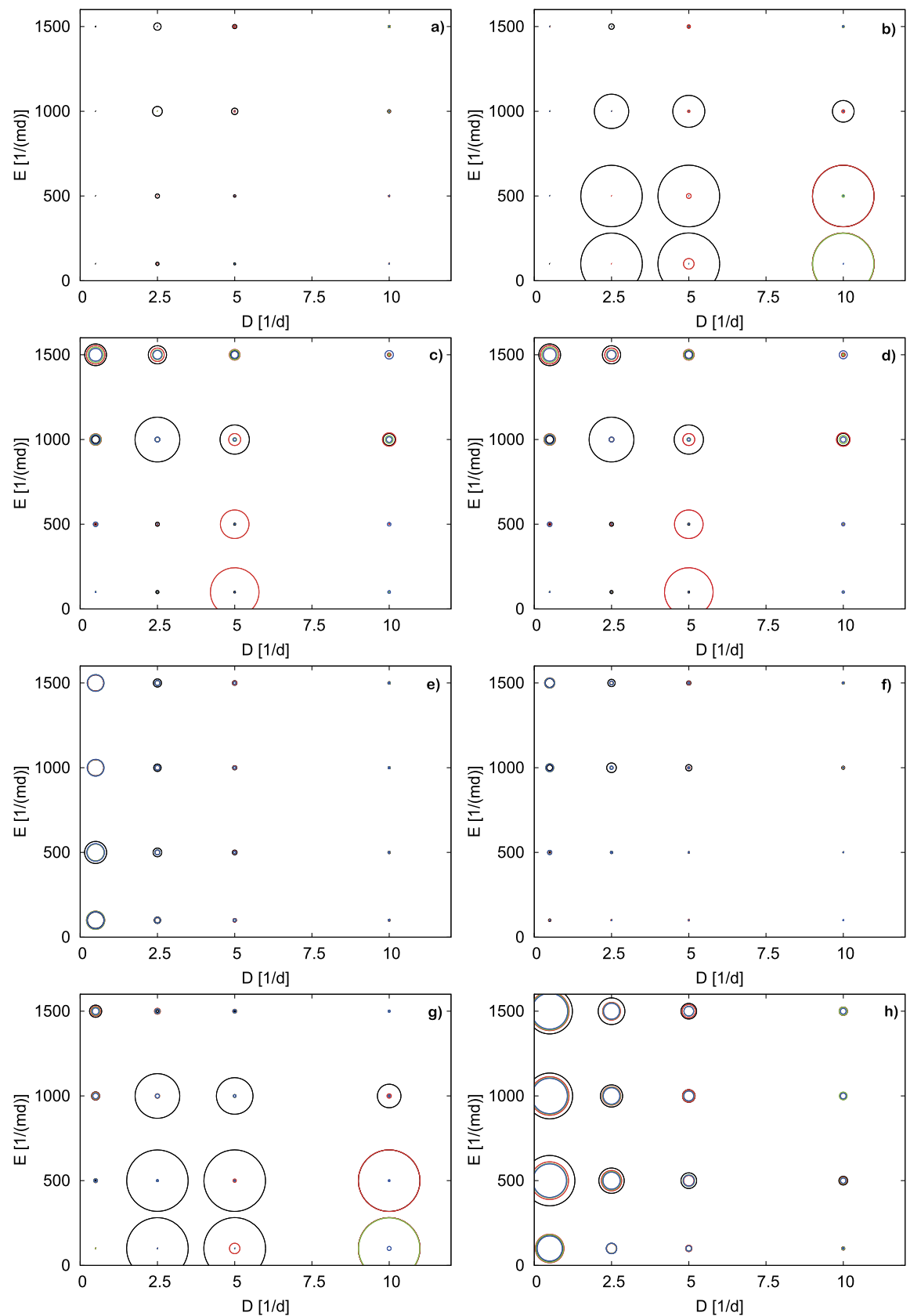

Fig. 7 Relative differences between the hybrid model and the biofilm-only model at steady state for dilution rates $D=0.5,2.5,5,10$ (x-axis), erosion rates $E=100,500,1000,1500$ (y-axis) and amounts of suspended carriers $c=0,50,100,150$ shown in order in colors black, red, green, blue. The unit for the circles is the same as the x-axis, i.e. a difference of 1 equals a difference of $100 \%$. The studied quantities are: (a) ammonium removal, (b) nitrate production, (c) nitrite concentration in reactor, (d) nitrite net production, (e) biofilm thickness, (f) $\mathrm{AOB},(\mathbf{g}) \mathrm{NOB}$, (h) inerts in biofilm (Color figure online) 
green, and $c=150$ blue. The circle radii are scaled with respect to the unit of the $D$ axis. We note in some cases (nitrate production in Fig. 7b and amount of NOB in biofilm in Fig. $7 \mathrm{~g}$, for low $E$ ) relative differences between the models of $100 \%$. In these cases, the quantity of interest is close to 0 in both models. For example, the steady state value of nitrate production in the hybrid model for the parameter combination $D=2.5, E=500, c=0$ was $0.00163 \mathrm{~g} /$ day and in the biofilm-only model $0.00000947 \mathrm{~g} /$ day. The NOB values for the same parameter combination were $0.000221 \mathrm{~g}$ in the hybrid model and $0.00000146 \mathrm{~g}$ in the biofilm-only model. Note that the values from the hybrid model are given as averages over $\alpha$; see Eq. (20). A different case with the combination of parameters $D=10, E=100, c=100$ resulted in nitrate production of $0.0335 \mathrm{~g} /$ day in the hybrid model, $0.000319 \mathrm{~g} /$ day in the biofilm-only model, and an amount of NOB $0.00429 \mathrm{~g}$ in the hybrid and $0.0000419 \mathrm{~g}$ in the biofilm-only model. Thus, in absolute terms the data agree well. These cases aside, the largest relative differences can be found in $7 \mathrm{c}, \mathrm{d}, \mathrm{h}$ for the nitrite concentration and net production along with biofilm inert biomass.

The smallest differences can be found in Figs. 7a, f for ammonium removal and biofilm AOB biomass with small circles for all combinations of parameters. These two variables are connected to each other through the consumption of ammonium by $\mathrm{AOB}$, which constitutes the first and main reaction in two-step nitrification. As long as oxygen is available, it is a straightforward reaction. Although suspended biomass contributes to the ammonium removal, the contribution is not significantly different than what is obtained by the biofilm in the biofilm-only model.

Only in some cases do the differences between the models change monotonically with the parameters. For example, in Figs. 7e, h the differences in biofilm thickness and inert biomass get smaller with increasing $D$ due to washout of suspended biomass and domination of biofilm in the hybrid model. However, generally such monotonic correlations cannot be confirmed, cf. Figs. 7a, c, d, f, g.

It has been suggested by, e.g. Manz et al. (2005) that the detachment rate should increase with increasing flow rate. Although this is not explicitly included in the standard biofilm detachment model that we use here, the diagonals in Figs. 7a-h correspond to the case where the detachment rate increases with the flow rate. In Figs. 7e, $\mathrm{h}$ we see how the differences between both models become smaller as $D$ increases. For all other cases, no monotonic relationship is observed; for most quantities of interests the differences between the models along this $D-E$ diagonal are largest for $D=5, E=1000$.

In summary, the influence and interplay of $\alpha, c, D, E$ with respect to the quantities of interest is complex. It cannot be inferred a priori whether for a given parameter set the biofilm-only model gives a good approximation of the more complex hybrid model. However, it can be expected that some variables, e.g. nitrite concentration and amount of NOB biomass (i.e. the variables primarily associated with the second step of nitrification), are more likely to be affected by changes in the four parameters. Other variables, like ammonium concentration and amount of AOB biomass (i.e. the parameters primarily associated with the first step in nitrification), tend to be more robust and not so strongly affected. 
4.6 How Well Is the Microbially Complex System Studied Here Described Qualitatively by the Much Simpler Previously Studied Single-Species Model?

In Mašić and Eberl (2012), we investigated with analytical and computational techniques a simple hybrid biofilm-suspended model for a single species, single substrate system. While of course such a simpler model will never be able to describe a more complex system, like the nitrification system, quantitatively, we can find certain qualitative similarities. In particular, the results concerning the primary process, ammonium removal, and $\mathrm{AOB}$ resemble closely those found in the simpler model. The same holds true for the effect of changes in the detachment rate. Furthermore, while we are not able to provide rigorous proof in the case of the multi-species system, it appears from the results in Appendix B that the stability and instability of the trivial equilibrium (washout of biomass from the reactor) is qualitatively similar to the results of the single species model. That said, simpler single species models that are at least to some extent analytically tractable can also provide some useful insight in microbially more complex systems and, therefore, should have their place in biofilm modeling.

\section{Conclusions}

The nitrifying system that is the subject of this paper is generally considered a biofilm system. Nevertheless, due to detachment of biomass from the biofilm surface, suspended biomass is always present in the bulk liquid. In our study, we aimed to investigate and quantify the role of the suspended biomass in this predominantly biofilm system. Intuitively, the relative contribution of suspended biomass to nutrient removal is higher at low dilution rates in contrast to large dilution rates when biomass washout is increased. On the other hand, a large dilution rate improves the biofilm performance due to an increased nutrient load. Furthermore, in a completely mixed tank reactor the suspended biomass experiences higher substrate concentrations than biomass located in the inner layers of the biofilm, wherefore it can be expected to be more effective in removal. Moreover, the amount of colonizable surface area affects biofilm development and its contribution to nutrient removal. Taking these effects together, an a priori prediction of the role of suspended biomass for overall reactor performance and intermittent processes is difficult to make. To investigate this complex system in detail, numerous simulation experiments were conducted. We reached the following conclusions:

- Increased dilution rate increases the steady state values of all variables except nitrate effluent concentration and amount of suspended biomass. Increased colonizable surface area increases the steady state values of all variables, except ammonium effluent concentration, biofilm thickness and net production of nitrite, which are decreased instead.

- The contribution of suspended biomass can be significant in the intermediate steps in nitrification, for NOB and for inert biomass; not accounting explicitly for suspended biomass leads to quantitatively different results. On the other hand, overall 
reactor performance in terms of ammonium removal and nitrate production is described well by a simpler model that does not explicitly account for suspended biomass, in which the role of suspended biomass is implicitly subsumed in the activity of the biofilm. However, suspended biomass always contributes to ammonium removal, at most for low dilution rates.

- With respect to the primary biomass group AOB and ammonium removal as the primary process of interest, and their dependence on reactor operating conditions, the qualitative behavior of the multi-species, multi-substrate nitrification model is reasonably well described by the qualitative behavior of a mathematically simpler single species, single substrate model, which is partially tractable with qualitative analytical techniques. Quantitative predictions, however, require numerical simulations of the essentially more complex multi-species system.

- Detachment has a large impact on the steady state values of the intermediate compounds and processes in the hybrid model, particularly on NOB biomass in the biofilm. Its impact on reactor performance, measured in terms of ammonium removal, is less pronounced. Moreover, the attachment rate $\alpha$ only plays a minor role in this respect.

- We compared a simpler biofilm-only model with the hybrid biofilm-suspended model and found that the qualitative agreement between both models can vary greatly for some parameter value combinations of dilution rates, colonizable surface areas, erosion parameters and (re-)attachment rates. However, we were not able to conclude simple qualitative correlations, such as monotonicity relationships, due to the biological, mathematical and numerical complexity of the model.

- Overall, we conclude from our simulations that a detailed mathematical model comprising biofilm and suspended biomass should be used if the details of the microbial process are in the focus of the investigation. If only coarse information regarding reactor behavior is required, such level of model complexity might not be necessary, but a simpler biofilm-only model might suffice. The dependent variables and quantities of interest primarily associated with the first step in nitrification are found to be rather insensitive with respect to operating conditions and description of the detachment process and also are well described by a simpler model that neglects suspended biomass. The variables primarily associated with the second step in nitrification, however, show much more sensitivity in this regard.

Acknowledgements This work was conducted while the first author was a Ph.D. student at Lund University (Sweden), AM acknowledges the support received from the Centre for Mathematical Sciences. HJE was supported in parts by NSERC of Canada with a Discovery Grant.

\section{Appendix A: Growth and Reaction Rates}

The growth rates $\mu_{A}, \mu_{N}, \mu_{I}$ are calculated from Table 1 through

$$
\mu_{i}=\sum_{j=1}^{6} \frac{v_{i j} \cdot P_{j}}{f_{i} \cdot \rho}, \quad i \in I_{b},
$$


where

$$
\begin{aligned}
& \mu_{A}(\bar{S})=\mu_{A, \max } \cdot \frac{S_{\mathrm{O} 2}}{K_{A, \mathrm{O} 2}+S_{\mathrm{O} 2}}\left(\frac{S_{\mathrm{NH} 4}}{K_{\mathrm{NH} 4}+S_{\mathrm{NH} 4}}-(1+\eta) \cdot b_{A}\right) \\
& \mu_{N}(\bar{S})=\mu_{N, \max } \cdot \frac{S_{\mathrm{O} 2}}{K_{N, \mathrm{O} 2}+S_{\mathrm{O} 2}}\left(\frac{S_{\mathrm{NO} 2}}{K_{\mathrm{NO} 2}+S_{\mathrm{NO} 2}}-(1+\eta) \cdot b_{N}\right) \\
& \mu_{I}(\bar{S})=\frac{1}{f_{I}} \cdot\left(f_{X I}+\eta\right)\left(f_{A} \cdot b_{A} \cdot \frac{S_{\mathrm{O} 2}}{K_{A, \mathrm{O} 2}+S_{\mathrm{O} 2}}+f_{N} \cdot b_{N} \cdot \frac{S_{\mathrm{O} 2}}{K_{N, \mathrm{O} 2}+S_{\mathrm{O} 2}}\right) .
\end{aligned}
$$

Similarly, the reaction rates $r_{\mathrm{O} 2}, r_{\mathrm{NH} 4}, r_{\mathrm{NO} 2}, r_{\mathrm{NO} 3}$ are calculated through

$$
r_{k}=\sum_{j=1}^{6} v_{k j} \cdot P_{j}, \quad k \in I_{S}
$$

where

$$
\begin{aligned}
r_{\mathrm{O} 2}\left(f_{A} \rho, f_{N} \rho, \bar{S}\right)= & \frac{3.43-Y_{A}}{Y_{A}} \cdot \mu_{A, \max } \cdot \frac{S_{\mathrm{O} 2}}{K_{A, \mathrm{O} 2}+S_{\mathrm{O} 2}} \cdot \frac{S_{\mathrm{NH} 4}}{K_{\mathrm{NH} 4}+S_{\mathrm{NH} 4}} \cdot f_{A} \rho \\
& +\left(1-f_{X I}\right) \cdot b_{A} \cdot \frac{S_{\mathrm{O} 2}}{K_{A, \mathrm{O} 2}+S_{\mathrm{O} 2}} \cdot f_{A} \rho \\
& +\frac{1.14-Y_{N}}{Y_{N}} \cdot \mu_{N, \max } \cdot \frac{S_{\mathrm{O} 2}}{K_{N, \mathrm{O} 2}+S_{\mathrm{O} 2}} \cdot \frac{S_{\mathrm{NO} 2}}{K_{\mathrm{NO} 2}+S_{\mathrm{NO} 2}} \cdot f_{N} \rho \\
& +\left(1-f_{X I}\right) \cdot b_{N} \cdot \frac{S_{\mathrm{O} 2}}{K_{N, \mathrm{O} 2}+S_{\mathrm{O} 2}} \cdot f_{N} \rho,
\end{aligned}
$$

$r_{\mathrm{NH} 4}\left(f_{A} \rho, f_{N} \rho, \bar{S}\right)=\left(\frac{1}{Y_{A}}+i_{A}\right) \cdot \mu_{A, \max } \cdot \frac{S_{\mathrm{O} 2}}{K_{A, \mathrm{O} 2}+S_{\mathrm{O} 2}} \cdot \frac{S_{\mathrm{NH} 4}}{K_{\mathrm{NH} 4}+S_{\mathrm{NH} 4}} \cdot f_{A} \rho$

$$
\begin{aligned}
& -\left(i_{A}-i_{I} f_{X I}\right) \cdot b_{A} \cdot \frac{S_{\mathrm{O} 2}}{K_{A, \mathrm{O} 2}+S_{\mathrm{O} 2}} \cdot f_{A} \rho \\
& +i_{A} \cdot \mu_{N, \max } \cdot \frac{S_{\mathrm{O} 2}}{K_{N, \mathrm{O} 2}+S_{\mathrm{O} 2}} \cdot \frac{S_{\mathrm{NO} 2}}{K_{\mathrm{NO} 2}+S_{\mathrm{NO} 2}} \cdot f_{N} \rho \\
& -\left(i_{A}-i_{I} f_{X I}\right) \cdot b_{N} \cdot \frac{S_{\mathrm{O} 2}}{K_{N, \mathrm{O} 2}+S_{\mathrm{O} 2}} \cdot f_{N} \rho,
\end{aligned}
$$

$$
\begin{aligned}
r_{\mathrm{NO} 2}\left(f_{A} \rho, f_{N} \rho, \bar{S}\right)= & \left(-\frac{1}{Y_{A}}\right) \cdot \mu_{A, \max } \cdot \frac{S_{\mathrm{O} 2}}{K_{A, \mathrm{O} 2}+S_{\mathrm{O} 2}} \cdot \frac{S_{\mathrm{NH} 4}}{K_{\mathrm{NH} 4}+S_{\mathrm{NH} 4}} \cdot f_{A} \rho \\
& +\frac{1}{Y_{N}} \cdot \mu_{N, \max } \cdot \frac{S_{\mathrm{O} 2}}{K_{N, \mathrm{O} 2}+S_{\mathrm{O} 2}} \cdot \frac{S_{\mathrm{NO} 2}}{K_{\mathrm{NO} 2}+S_{\mathrm{NO} 2}} \cdot f_{N} \rho, \\
r_{\mathrm{NO} 3}\left(f_{N} \rho, \bar{S}\right)= & \left(-\frac{1}{Y_{N}}\right) \cdot \mu_{N, \max } \cdot \frac{S_{\mathrm{O} 2}}{K_{N, \mathrm{O} 2}+S_{\mathrm{O} 2}} \cdot \frac{S_{\mathrm{NO} 2}}{K_{\mathrm{NO} 2}+S_{\mathrm{NO} 2}} f_{N} \rho .
\end{aligned}
$$


Note that the growth rates as well as the reaction rates throughout the paper use both $\bar{C}(z)$ and $\bar{S}(t)$ as arguments. Furthermore, the reaction rates use $f_{A}(z, t) \rho, f_{N}(z, t) \rho$, and $u_{A}(t), u_{N}(t)$ as arguments.

\section{Appendix B: Remarks on Equilibria}

\section{B.1 Washout Equilibrium}

It can be verified that the trivial equilibrium with $\lambda=u_{A}=u_{N}=u_{I}=0$ and bulk substrate concentrations attaining inflow concentrations always exists. To investigate the stability of this equilibrium in dependence of model parameters with analytical techniques turns out to be rather difficult because of the involved hybrid structure of the mathematical model. For the simpler single-species model of a biofilm reactor with suspended bacteria, it was found in Mašić and Eberl (2012) that two conditions need to be satisfied for the trivial or washout equilibrium to be stable: (i) The dilution rate needs to be larger than the growth rate of the suspended bacteria. This is the standard criterion for washout in completely mixed continuous reactors. (ii) In order to avoid also that a biofilm can establish itself, the bulk substrate concentration must be small enough so that the diffusive flux of substrate into the biofilm is smaller than the decay rate. Both conditions together lead to the observation that a high flow rate alone is not sufficient to lead to washout of the microbiology from the reactor. This also explains to some extent why bacterial biofilms are very difficult to eradicate and to prevent in situations where they are unwanted.

In the absence of analytical results, numerical simulations are carried out to investigate whether the observations from the single species biofilm model carry over to the essentially more involved multi-species model. The simulations start with initial data that are small perturbations of the trivial equilibrium. In particular, we introduce a small amount of biomass in the system, both suspended and sessile, namely

$$
\begin{aligned}
& \lambda(0)=1 \mu \mathrm{m}, \quad f_{A}(0)=f_{N}(0)=0.5, \quad f_{I}(0)=0, \\
& u_{A}(0)=u_{N}(0)=2 \cdot 10^{-6} \mathrm{~g}, \quad u_{I}(0)=0 \mathrm{~g} .
\end{aligned}
$$

The simulation parameters are as in Tables 3 and 2. We set the dilution rate to $D=5 /$ day, larger than the maximum specific growth rate of both species. The colonizable surface area is set to $A=A_{\text {reactor }}$, i.e. we consider a reactor without added biofilm carriers. In our illustrative studies, we investigate the stability of the system with respect to the inflow concentration of ammonium $S_{\mathrm{NH} 4}^{0}$, assuming that neither nitrite nor nitrate are present in the influent, $S_{\mathrm{NO} 2}^{0}=S_{\mathrm{NO} 3}^{0}=0 \mathrm{~g} / \mathrm{m}^{3}$. The bulk oxygen concentration is kept at $S_{\mathrm{O} 2}=5 \mathrm{~g} / \mathrm{m}^{3}$.

We increase the inflow concentration of ammonium, as the growth controlling substrate, in small increments, starting from $S_{\mathrm{NH} 4}^{0}=0$. In all cases, the system reaches steady state. In Fig. 8, we plot the amount of suspended biomass and the biofilm thickness at steady state. We find that the trivial equilibrium is stable for $S_{\mathrm{NH} 4}^{0}<0.04 \mathrm{~g} / \mathrm{m}^{3}$, i.e. no biomass can be established for very small substrate concentrations. When the inflow concentration of ammonium is further increased, the 


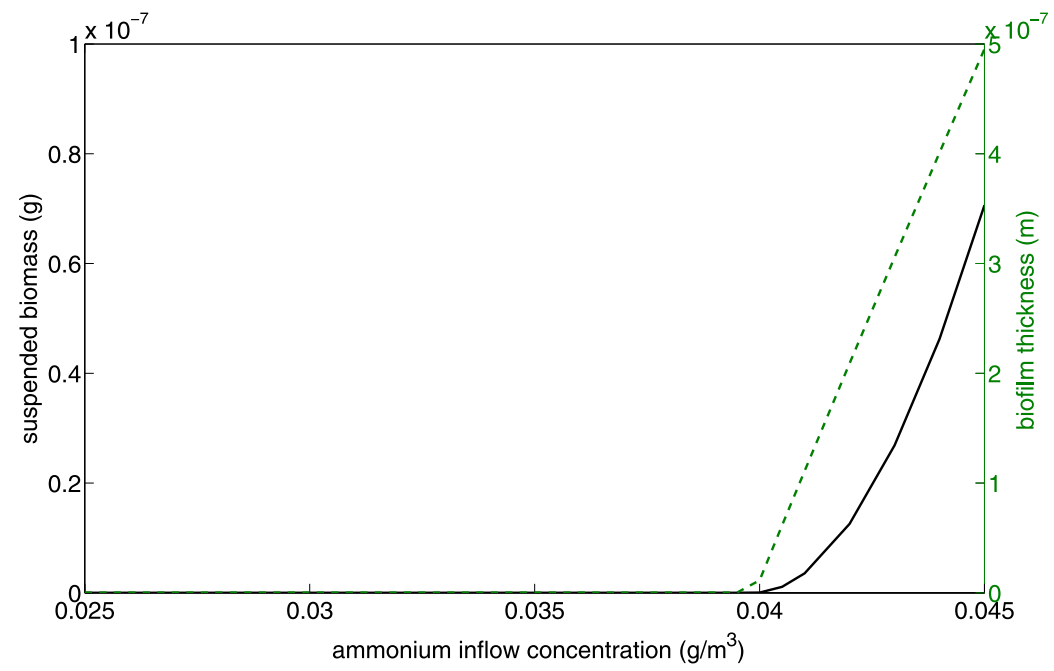

Fig. 8 Steady state values for varying the initial ammonium concentration $\mathrm{S}_{\mathrm{NH} 4}^{0}$ for total amount of suspended biomass [g] (solid, left y-axis) and biofilm thickness [m] (dashed, right y-axis) in the hybrid model at $D=5 /$ day and $A=A_{\text {reactor }}$ (Color figure online)

trivial equilibrium becomes unstable and both, biofilm and suspended populations establish themselves. The observed critical value at which the trivial equilibrium becomes unstable is much smaller than the half-saturation coefficient for ammonium $K_{\mathrm{NH} 4}=0.169 \mathrm{~g} / \mathrm{m}^{3}$, which implies that severe substrate limitation is required for stability of washout. Initially, the biofilm biomass increases faster than the suspended biomass, but both are always present in accordance with Remark 2.2.

In conclusion, the stability of the trivial equilibrium depends on the parameters and operating conditions. A biofilm cannot be established if the influent substrate concentration is too small.

We compare these findings for the nitrification model with the mathematically simpler single species single substrate model, for which the stability of the trivial equilibrium could be investigated with analytical techniques. First, we point out the qualitative similarity of Fig. 8 with Fig. 5 of Mašić and Eberl (2012). For a more quantitative comparison of the dual species system with four substrates with the single species, single substrate model, we recall that in Proposition 3.5 of Mašić and Eberl (2012) a necessary and sufficient condition for the stability of the trivial equilibrium was found. Derived from this in Corollary 3.6 were easier to use sufficient conditions for stability and for instability. Each of these two sufficient conditions consists of a pair of inequalities for the model parameters, one stemming from the suspended biomass and one from the biofilm. In essence, it was found for the single species, single substrate model that the trivial equilibrium is stable if growth of suspended biomass is dominated by washout, decay and attachment, and if production of new biomass in the biofilm is dominated by lysis. In particular, it was found that the stability of the trivial equilibrium is independent of the detachment coefficient $E$, in accordance with the findings of Abbas et al. (2012) for a biofilm reactor without suspended biomass. 
The simple stability criterion of Mašić and Eberl (2012) cannot be directly applied to or derived with rigorous techniques for the multi-species, multi-substrate case. Nevertheless, based on the biological interpretation of the stability criterion we can suggest an easy to evaluate "approximate" ad hoc criterion for the multi-species case, where we focus on the persistence of $\mathrm{AOB}$ as the main player in the system in dependence of ammonium and oxygen, as its primary substrates that are required for growth. Since the oxygen concentration in the aqueous phase is fixed, this ad hoc criterion is cast as a condition on the bulk concentration of ammonium. An important further adaptation is required due to the fact that in the simpler model of Mašić and Eberl (2012) biomass decay was described as a simple lysis model, while in the current nitrification model decay of active biomass is due to endogenous respiration and inactivation. Incorporating this, we adapt from Mašić and Eberl (2012) the following non-rigorous ad hoc criterion for instability of the trivial equilibrium by comparing growth and loss of AOB:

$$
\mu_{A, \max } \frac{S_{\mathrm{NH} 4}^{0}}{K_{\mathrm{NH} 4}+S_{\mathrm{NH} 4}^{0}} \frac{S_{\mathrm{O} 2}^{0}}{K_{A, \mathrm{O} 2}+S_{\mathrm{O} 2}^{0}}>D+\alpha+\frac{S_{\mathrm{O} 2}^{0}}{K_{A, \mathrm{O} 2}+S_{\mathrm{O} 2}^{0}} b_{A}(1+\eta)
$$

or

$$
\frac{S_{\mathrm{NH} 4}^{0}}{K_{\mathrm{NH} 4}+S_{\mathrm{NH} 4}^{0}}>\frac{b_{A}(1+\eta)}{\mu_{A}},
$$

where the first inequality stems from the persistence of suspended biomass and the second one from persistence of the biofilm. From this double inequality and the parameters in Tables 3 and 2, we can obtain the critical inflow concentration $S_{\mathrm{NH} 4}^{0}$ for which stability of the trivial equilibrium is lost as $S_{\mathrm{NH} 4}^{0} \approx 0.04$, in very good quantitative agreement with the more accurate simulation documented in Fig. 8. While this finding is not a formal proof, it suggests strongly that (i) the fate of AOB is critically determining the overall fate of the system, and (ii) that the qualitative behavior of the multi-species, multi-substrate system can be well approximated by the qualitative behavior of the simpler and mathematically easier accessible single species, single substrate model.

\section{B.2 Persistence Equilibrium}

For the much simpler Freter model that describes persistence of a single species depending on a single resource in a CSTR with wall attachment, it could be shown that at least one asymptotically stable non-trivial equilibrium exists (Jones et al. 2003). For the single species, single substrate biofilm model in Mašić and Eberl (2012) it was not possible to handle this question analytically, due to the essentially more complicated algebraic computations that would need to be performed. Nevertheless, it was confirmed in numerous numerical simulations that the system attains a nontrivial equilibrium if the trivial equilibrium is unstable. The current model is not only algebraically, but also structurally much more involved than the single species model and, therefore, we cannot expect to be able to investigate the question of existence and stability of non-trivial equilibria in dependence of model parameters mathematically. For the present study, the results of which are documented in the subsequent 
sections, extensive numerical simulations of the model have been conducted. In these simulations, the biological parameters were kept constant at values validated against experimental data and the parameters describing operating conditions for the reactor were varied. The model converged to an equilibrium in all cases.

\section{References}

Abbas, F., Sudarsan, R., \& Eberl, H. J. (2012). Longtime behaviour of one-dimensional biofilm models with shear dependent detachment rates. Math. Biosci. Eng., 9(2), 215-239.

Ballyk, M. M., Jones, D. A., \& Smith, H. L. (2001). Microbial competition in reactors with wall attachment. Microb. Ecol., 41(3), 210-221.

Boltz, J. P., Morgenroth, E., Brockmann, D., Bott, C., Gellner, W. J., \& Vanrolleghem, P. A. (2011). Systematic evaluation of biofilm models for engineering practice: components and critical assumptions. Water Sci. Technol., 64(4), 930-944.

Boltz, J. P., Johnson, B. R., Daigger, G. T., \& Sandino, J. (2009a). Modeling integrated fixed-film activated sludge and moving-bed biofilm reactor systems I: mathematical treatment and model development. Water Environ. Res., 81(6), 555-575.

Boltz, J. P., Johnson, B. R., Daigger, G. T., Sandino, J., \& Elenter, D. (2009b). Modeling integrated fixedfilm activated sludge and moving-bed biofilm reactor systems II: evaluation. Water Environ. Res., 81(6), 576-586.

Boltz, J. P., Daigger, G. T., Johnson, B. R., Hiatt, W., \& Grady, C. P. L. Jr. (2009c). Expanded process model describes biomass distribution, free-ammonia/nitrous acid inhibition and competition between ammonia oxidizing bacteria (AOB) and nitrite oxidizing bacteria (NOB) in submerged biofilm and integrated fixed film activated sludge (IFAS) bioreactors. In Proceedings WEF, nutrient removal (pp. 187-206).

Chaudhry, M. A. S., \& Beg, S. A. (1999). A review on the mathematical modeling of biofilm processes: advances in fundamental of biofilm modelling. Chem. Eng. Technol., 21(9), 701-710.

Desharnais, R. A. \& Yiqi, L. (Eds.) (2005). Population dynamics and laboratory ecology: 37 (Advances in ecological research) (1st ed.). San Diego: Elsevier.

Di Trapani, D., Mannina, G., Torregrossa, M., \& Viviani, G. (2010a). Comparison between hybrid moving bed biofilm reactor and activated sludge system: a pilot plant experiment. Water Sci. Technol., 61(4), 891-902.

Di Trapani, D., Mannina, G., Torregrossa, M., \& Viviani, G. (2010b). Quantification of kinetic parameters for heterotrophic bacteria via respirometry in a hybrid reactor. Water Sci. Technol., 61(7), 1757-1766.

Elenter, D., Milferstedt, K., Zhang, W., Hausner, M., \& Morgenroth, E. (2007). Influence of detachment on substrate removal and microbial ecology in a heterotrophic/autotrophic biofilm. Water Res., 41(20), 4657-4671.

Fgaier, H., \& Eberl, H. J. (2011). Antagonistic control of microbial pathogens under iron limitations by siderophore producing bacteria in a chemostat setup. J. Theor. Biol., 273(1), 103-114.

Fouad, M., \& Bhargava, R. (2005). A simplified model for the steady-state biofilm-activated sludge reactor. J. Environ. Manag., 74(3), 245-253.

Freter, R., Brickner, H., Fekete, J., Vickerman, M., \& Carey, K. (1983). Survival and implantation of Escherichia coli in the intestinal tract. Infect. Immun., 39, 686-703.

Gray, N. F. (2004). Biology of wastewater treatment (2nd ed.). London: Imperial College Press.

Hunik, J. H., Tramper, J., \& Wijffels, R. H. (1994). A strategy to scale up nitrification processes with immobilized cells of nitrosomonas europaea and nitrobacter agilis. Bioprocess Biosyst. Eng., 11(2), 73-82.

Hellinga, C., Schellen, A. A. J. C., Mulder, J. W., van Loosdrecht, M. C. M., \& Heijnen, J. J. (1998). The SHARON process: an innovative method for nitrogen removal from ammonium-rich waste water. Water Sci. Technol., 37(9), 135-142.

Johnson, T. L., McQuarrie, J. P., \& Shaw, A. R. (2004). Integrated Fixed-film Activated Sludge (IFAS): the new choice for nitrogen removal upgrades in the United States. In Proceedings WEFTEC, session 1-10 (pp. 296-318).

Jones, D., Kojouharov, H. V., Le, D., \& Smith, H. (2003). The Freter model: a simple model of biofilm formation. J. Math. Biol., 47(2), 137-152. 
Kim, H., Pei, R., Boltz, J. P., Gellner, J., Gunsch, C., Freudenberg, R. G., Dodson, R., \& Schuler, A. J. (2009). Nitrification and AOB/NOB populations in integrated fixed-film activated sludge: measurements and modeling. In Proceedings WEF, nutrient removal (pp. 84-96).

Mannina, G., Di Trapani, D., Viviani, G., \& Ødegaard, H. (2011). Modelling and dynamic simulation of hybrid moving bed biofilm reactors: model concepts and application to a pilot plant. Biochem. Eng. J., 56(1-2), 23-36.

Mašić, A. (2013). Investigation of a biofilm reactor model with suspended biomass. Ph.D. Thesis, Lund University, Sweden.

Mašić, A., \& Eberl, H. (2012). Persistence in a single species CSTR model with suspended flocs and wall attached biofilms. Bull. Math. Biol., 74(4), 1001-1026.

Mašić, A., Bengtsson, J., \& Christensson, M. (2010). Measuring and modeling the oxygen profile in a nitrifying moving bed biofilm reactor. Math. Biosci., 227(1), 1-11.

The Mathworks (2012). MATLAB online documentation. http://www.mathworks.com/help/techdoc/ref/ (Accessed on August 3).

Manz, B., Volke, F., Goll, D., \& Horn, H. (2005). Investigation of biofilm structure, flow patterns and detachment with magnetic resonance imaging. Water Sci. Technol., 52(7), 1-6.

Mazenc, F., \& Malisoff, M. (2012). Stability and stabilization for models of chemostats with multiple limiting substrates. J. Biol. Dyn., 6(2), 612-627.

Morgenroth, E., van Loosdrecht, M. C. M., \& Wanner, O. (2000). Biofilm models for the practitioner. Water Sci. Technol., 41(4-5), 509-512.

Northcott, K., Imran, M., \& Wolkowicz, G. S. K. (2012). Competition in the presence of a virus in an aquatic system: an SIS model in the chemostat. J. Math. Biol., 64(6), 1043-1086.

Pai, T.-Y. (2007). Modeling nitrite and nitrate variations in $\mathrm{A}^{2} \mathrm{O}$ process under different return oxic mixed liquid using an extended model. Process Biochem., 42(6), 978-987.

Peña-Miller, R., Lähnemann, D., Schulenburg, H., Ackermann, M., \& Beardmore, R. (2012). Selecting against antibiotic-resistant pathogens: optimal treatment in the presence of commensal bacteria. Bull. Math. Biol., 74, 908-934.

Picioreanu, C., Kreft, J.-U., \& van Loosdrecht, M. C. (2004). Particle-based multidimensional multispecies biofilm model. Appl. Environ. Microbiol., 70(5), 3024-3040.

Rittmann, B. E., \& McCarty, P. L. (2001). Environmental biotechnology: principles and applications. New York: McGraw-Hill.

Salem, S., Moussa, M., \& van Loosdrecht, M. C. (2006). Determination of the decay rate of nitrifying bacteria. Biotechnol. Bioeng., 94(2), 252-262.

Sen, D., \& Randall, C. W. (2008a). Improved computational model (AQUIFAS) for activated sludge, integrated fixed-film activated sludge, and moving-bed biofilm reactor systems, part I: semi-empirical model development. Water Environ. Res., 80(5), 439-453.

Sen, D., \& Randall, C. W. (2008b). Improved computational model (AQUIFAS) for activated sludge, integrated fixed-film activated sludge, and moving-bed biofilm reactor systems, part II: multilayer biofilm diffusional model. Water Environ. Res., 80(7), 624-632.

Smith, H. L., \& Waltman, P. (1995). The theory of the chemostat. Cambridge: Cambridge University Press.

Smith, H. L., \& Thieme, H. R. (2012). Persistence of bacteria and phages in a chemostat. J. Math. Biol., 64(4), 951-979.

Stemmons, E. D., \& Smith, H. L. (2000). Competition in a chemostat with wall attachment. SIAM J. Appl. Math., 61(2), 567-595.

Szomolay, B., Klapper, I., \& Dindos, M. (2010). Analysis of adaptive response to dosing protocols for biofilm control. SIAM J. Appl. Math., 70(8), 3175-3202.

Szomolay, B. (2008). Analysis of a moving boundary value problem arising in biofilm modelling. Math. Methods Appl. Sci., 31(15), 1835-1859.

Thalla, A. K., Bhargava, R., \& Kumar, P. (2009). Design of multistage activated sludge-biofilm reactors: a rational approach. World Rev. Sci. Technol. Sustain. Dev., 6(2-4), 115-126.

Thalla, A. K., Bhargava, R., \& Kumar, P. (2010). Nitrification kinetics of activated sludge-biofilm system: a mathematical model. Bioresour. Technol., 101(15), 5827-5835.

Villadsen, J., Nielsen, J., \& Lidén, G. (2011). Bioreaction engineering principles (3rd ed.). New York: Springer.

Wanner, O., Eberl, H., Morgenroth, E., Noguera, D. R., Picioreanu, C., Rittmann, B., \& van Loosdrecht, M. (2006). Mathematical modeling of biofilms (Scientific and Technical Report No. 18). IWA Publishing.

Wanner, O., \& Gujer, W. (1986). A multispecies biofilm model. Biotechnol. Bioeng., 28(3), 314-328. 
Wiesmann, U. (1994). Biological nitrogen removal from wastewater. Adv. Biochem. Eng. Biotechnol., 51, $113-154$

Wik, T. (1999). On modeling the dynamics of fixed biofilm reactors. Ph.D. Thesis, Chalmers University of Technology, Goteborg, Sweden.

Wyffels, S., van Hulle, S. W., Boeckx, P., Volcke, E. I., van Cleemput, O., Vanrolleghem, P. A., \& Verstraete, W. (2004). Modeling and simulation of oxygen-limited partial nitritation in a membraneassisted bioreactor (MBR). Biotechnol. Bioeng., 86(5), 531-542. 